\title{
A Self-adaptive Algorithm for Topology Matching in Unstructured Peer-to-Peer Networks
}

\author{
Ali Mohammad Saghiri ${ }^{1} \cdot$ Mohammad Reza Meybodi $^{1}$
}

Received: 10 May 2014/ Accepted: 8 September 2015

(C) Springer Science+Business Media New York 2015

\begin{abstract}
Peer-to-peer networks are overlay networks that are constructed over underlay networks. These networks can be structured or unstructured. In these networks, peers choose their neighbors without considering underlay positions, and therefore, the resultant overlay network may have a large number of mismatched paths. In a mismatched path, a message may meet an underlay position several times, which causes redundant network traffic and end-to-end delay. In some of the topology matching algorithms called the heuristic algorithms, each peer uses a local search operator for gathering information about the neighbors of that peer located in its neighborhood radius. In these algorithms, each peer also uses a local operator for changing the connections among the peers. These matching algorithms suffer from two problems; neither the neighborhood radius nor the local operator can adapt themselves to the dynamicity of the network. In this paper, a topology matching algorithm that uses learning automata to adapt the neighborhood radius and an adaptation mechanism inspired from the Schelling segregation model to manage the execution of the local operator is proposed. To evaluate the proposed algorithm, computer simulations were conducted and then the results were compared with the results obtained for other existing algorithms. Simulation results have shown that the proposed algorithm outperforms the existing algorithms with respect to end-toend delay and number of mismatched paths.
\end{abstract}

Keywords Mismatched paths - Local search - Local operator - Schelling segregation model $\cdot$ Learning automata

Mohammad Reza Meybodi

mmeybodi@aut.ac.ir

Ali Mohammad Saghiri

a_m_saghiri@aut.ac.ir

1 Soft Computing Laboratory, Computer Engineering and Information Technology Department, Amirkabir University of Technology (Tehran Polytechnic), 424 Hafez Ave., Tehran, Iran 


\section{Introduction}

Peer-to-peer networks are computer networks in which all peers have the same role (client and server). In these networks, all peers communicate directly with each other. These networks are overlay networks that are constructed over underlay networks. There are two different types for peer-to-peer networks: structured and unstructured. In unstructured peer-to-peer networks such as Gnutella [1], GIA [2], and Freenet [3], there are some lightweight algorithms to manage the overlay topology. Unstructured peer-to-peer networks are widely used because their design are simple. In structured peer-to-peer networks such as Chord [4], and CAN [5], distributed algorithms are provided to manage the overlay topology that can provide efficient resource locating algorithms. In structured peer-to-peer networks, any resource can be located within a bounded number of hops. A major consequence of the network dynamicity caused by joining and leaving peers in both structured and unstructured peer-to-peer networks is that the overlay topology can change drastically over time. The network changes might adversely affect the performance of the overlay topology in terms of traffic and end-to-end delay. This issue is more important in an unstructured peer-to-peer network because in such a network, peer dynamics can rapidly change an initially good topology to an ineffective one. To prevent the performance degradations caused by the dynamicity of peer-to-peer networks, the topology management algorithms should be able to adapt themselves to the dynamic conditions of the network [1]. In unstructured peer-to-peer networks, for designing the topology management algorithms several problems such as topology mismatching [6-9] and topology clustering [10] must be solved. Since the primary goal of this paper is to design an adaptive algorithm for topology mismatch problems in peer-to-peer networks, the rest of the introduction section will be devoted to a more detailed discussion about this problem.

Consider $n$ peers that are connected to each other through an overlay network over an underlay network. The topology of the overlay network can be represented by an overlay graph $G=(V, E)$ where $V=\left\{\right.$ peer $_{1}$, peer $_{2}, \ldots$, peer $\left._{n}\right\}$ is a set of peers and $E \subseteq V \times V$ is a set of links connecting the peers. The topology of the underlay network can be also represented by an underlay graph $G^{\prime}=\left(V^{\prime}, E^{\prime}\right)$ where

Fig. 1 An example of topology mismatch problem

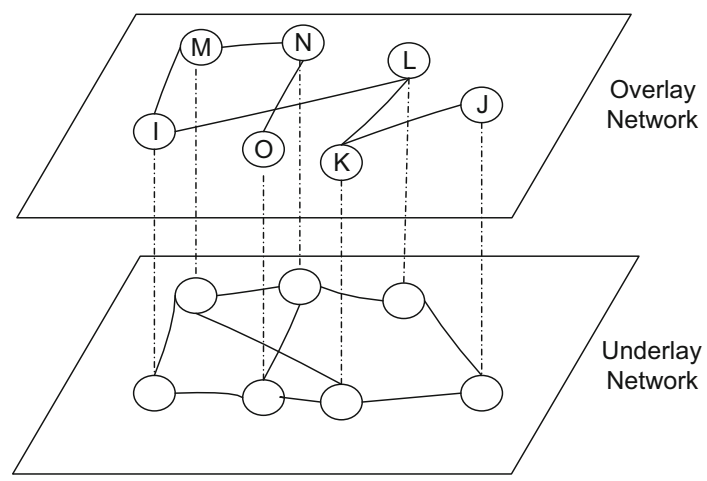


$V^{\prime}=\left\{\right.$ position $_{1}$, position $_{2}, \ldots$, position $\left._{n}\right\}$ is the set of positions in the underlay network and $E^{\prime} \subseteq V^{\prime} \times V^{\prime}$ is a set of links connecting the positions. In the peer-topeer network each peer in $V$ is mapped to a position in $V^{\prime}$ according to a one-to-one function $H: V \rightarrow V^{\prime}$. In both structured peer-to-peer networks and unstructured peer-to-peer networks, peers choose their neighbors without considering underlay positions and for this reason, the topology of the overlay network may have been mismatched with the topology of the underlay network. Creating a large number of mismatched paths is one of the problems that topology mismatching causes. Figure 1 shows an example of a mismatched path. To transfer a message from $L$ to $J(L \rightarrow J)$ in the overlay network path $L \rightarrow K \rightarrow J$ is used, but in the underlay network, we may have an inefficient path such as $(L \rightarrow J \rightarrow K \rightarrow J)$. This means that a message may meet an underlay position several times that causes the communication delay and traffic of flooding techniques to increase. In this example, path $(L \rightarrow J)$ is called a mismatched path. Since the mismatched paths cause redundant end-to-end delays, a type of topology matching algorithm, such as those reported in $[6,7,11,12]$ try to reconfigure the overlay network using local operators in order to decrease redundant delays caused by the mismatched paths. Therefore, the sum of end-to-end delays of the overlay network computed using (1) is used as an objective function that should be minimized by the matching algorithms.

$$
M(G, H)=\sum_{p e e r_{i} \in V} \sum_{\text {peer }_{j} \in V-\left\{\text { peer }_{i}\right\}} I(E,(i, j)) \times d_{i j}
$$

In (1), $d_{i j}=D\left(H\left(\right.\right.$ peer $\left._{i}\right), H\left(\right.$ peer $\left.\left._{j}\right)\right)$ is the end-to-end delay from peer $r_{i}$ to peer $_{j}$, function $D: V^{\prime} \times V^{\prime} \rightarrow \mathbb{R}$ computes the end-to-end delay between the pairs of positions in the underlay network, function $H: V \rightarrow V^{\prime}$ maps a peer in the overlay network into a position in the underlay network and function $I$ is an indicator function that takes a set of links $\mathrm{E}$ and a link $(i, j)$ and returns 1 if link $(i, j)$ is in $\mathrm{E}$ and 0 otherwise.

In some of the topology matching algorithms, each peer uses a local search operator for gathering information about the neighbors of that peer located in its neighborhood radius (in the overlay network). In these algorithms each peer also uses a local operator for changing the connections among the peers with the aim of decreasing the number of mismatched paths of the overlay. This type of algorithm is used in both structured $[13,14,15]$ and unstructured peer-to-peer networks $[6,7$, 16]. These algorithms reconfigure a given overlay graph $G^{f}=\left(V, E^{f}\right)$ to another graph $G^{o}=\left(V, E^{o}\right)$ in such a way that $M(G, H)$ (as given in (1)) is minimized. Let $\mathcal{L}$ be the set of all of graphs that can be used as topology for the overlay network, and $\mathrm{deg}_{i}$ denotes the number of links of peer $_{i}$ when the graph of the overlay is $G$. Both the $P R O P-O$ algorithm reported in $[6,15]$, and the algorithm proposed in this paper try to find an overlay graph so that (2) is minimized subject to constraint (3).

$$
\min _{G \in \mathcal{L}} \sum_{\text {peer }_{i} \in V} \sum_{\text {peer }_{j} \in V-\left\{\text { peer }_{i}\right\}} I(E,(i, j)) \times d_{i j}
$$




$$
\text { s.t } \quad \operatorname{deg}_{i}=\operatorname{deg}_{i}^{f} \quad \forall_{\text {peer }}
$$

where $\operatorname{deg}_{i}^{f}$ denotes the number of links of peer $_{i}$ when the graph of the overlay is $G^{f}$. Constraint (3) means that the matching algorithm should not change the degree of peers. This constraint tries to hold some characteristics of the graph of the overlay network such as degree distribution and number of links. Changing the degree of peers is not appropriate in both unstructured networks and structured networks, and therefore, satisfying the constraint (3) enables the topology matching algorithm to be used in a wide range of peer-to-peer networks. To satisfy constraint (3), a local operator called the exchange operator is proposed in [6]. Figure 2 shows how the exchange operator exchanges an equal number of neighbors between peer $_{i}$ and peer $_{j}$ if $d_{k i}+d_{l j}>d_{k j}+d_{l i}$. In this operator, the edges $\left(\right.$ peer $_{i}$, peer $\left._{k}\right)$ and $\left(\right.$ peer $_{j}$, peer $\left._{l}\right)$ will be replaced by $\left(\right.$ peer $_{j}$, peer $\left._{k}\right)$ and $\left(\right.$ peer $_{i}$, peer $\left._{l}\right)$ only if peer ${ }_{i}$ and peer $_{j}$ are adjacent to each other. With variable neighborhood radii, the exchange operator can be extended if there is a path between peer $_{i}$ and peer ${ }_{j}$. In this paper, $\left\{\right.$ peer $_{i}$, peer $\left._{j}\right\}$ and $\left\{\right.$ peer $_{k}$, peer $\left._{l}\right\}$ are called corresponding peers and candidate peers, respectively. Note that the exchange operator does not change the number of connections of the peers of the network during the exchange operation.

Existing matching algorithms such as $X$-BOT (Bias the Overlay Topology according to criteria X) [12, 17], Two-Hop-Away Neighbor Comparison and Selection (THANCS) [7], and Peer-exchange Routing Optimizing Protocol (PROP) $[6,15]$ suffer from two problems. The first problem is that there is no adaptive mechanism for setting the neighborhood radius parameter. Finding an appropriate value for this parameter manually is a time consuming process and also error prone. Large neighborhood radii speed up the convergence of the matching algorithm (because the number of candidate peers at each step increases), but it increases the number of exchanges that must be endured until the convergence of the algorithm. Also, large neighborhood radii cause higher traffic and computational overhead of the network. Small neighborhood radii decreases the number of candidate peers at each step of the algorithm, which causes the number of exchanges to be increased. Small neighborhood radii result in lower traffic and computational overhead. Because of the dynamicity of peer-to-peer networks, the operational environment and the neighbors of each peer may change over time (peers continually join and leave the network) and for this reason, using a fixed neighborhood radius for the operation of the local search operation may not be appropriate. The second problem is the lack of an adaptive mechanism for managing the execution of the local operator of the marching algorithm. A non-adaptive mechanism for managing the execution of the local operator leads to performing unnecessary local operations

Fig. 2 Exchange Operator
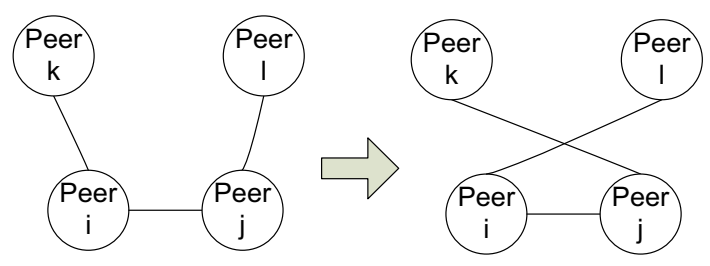
(such as exchange operations) that result in increasing the overhead of the matching algorithm (higher number of peers to be reconfigured and extra control messages). It should be noted that the structure of the underlay network is fixed, but the structure of the overlay network continually changes because of the network dynamicity caused by joining and leaving peers. Therefore the topology matching algorithms try to continually observe the changes occurring in the overlay network and modify the links of the overlay network in order to mitigate the negative effects of the topology mismatching problem. Therefore, having a self-adaptive mechanism for topology matching has a positive impact on the efficiency of a matching process.

Since peer-to-peer networks are large and also dynamic, algorithms for managing them must be self-organized algorithms, and for this reason several self-organized algorithms such as Ant Colony (ACO), Growing Neural Gas (GNG), and the Schelling Segregation Model (SSM) have been reported for topology management in the literature [10, 18-20]. The SSM, which has been used as part of the proposed algorithm is briefly discussed in the rest of this paragraph. The SSM is composed of independent and identical agents [21]. Each agent cares only about the composition of its own local neighborhood. Each agent using a function (called similarity function) calculates the portion of its neighbors which they have similar attributes with that agent. According to a rule called the happiness rule, each agent decides whether or not to change its neighbors. If the value of the similarity function is lower than a threshold $z$, the agent is unhappy and prefers to change its neighbors in order to increase the number of similar neighbors. This process continues until no agent wants to change its neighbors. In this model, designing a proper happiness rule is very crucial to the proper functioning of SSM.

In this paper, a self-adaptive algorithm for solving the topology mismatch problem in unstructured peer-to-peer networks will be proposed. In the proposed algorithm, we use learning automata to adapt the neighborhood radius and an adaptation mechanism inspired from the SSM to manage the execution of the exchange operator. To show the superiority of the proposed algorithm, computer experiments have been conducted and then the results are compared with the results obtained for algorithms $P R O P-O$ (an optimized version of $P R O P$ algorithm) [6, 15], $X$-BOT [12, 17], and THANCS [7]. The rest of this paper is organized as follows. In Sect. 2, the theory of learning automata is briefly described. Section 3 reviews the related works. In Sect. 4, we present a novel topology matching algorithm. Section 5 reports the results of experimentations and Sect. 6 concludes the paper.

\section{Learning Automata}

Learning automata (LAs) are models for adaptive decision making in random environments. A learning automaton has a finite set of actions. The learning process of the learning automaton is described as follows. Each time the learning automaton interacts with its environment, it randomly selects an action based on a probability vector. Initially, each action can be selected with equal probability. According to the response of the environment (reward or penalty) to the selected action, the learning automaton updates its action probability vector and then the procedure is repeated. 
The updating algorithm for the action probability vector is called the learning algorithm or the reinforcement scheme. If this algorithm is properly designated, the selection probability of the appropriate action approaches unity while the probabilities of other actions approach zero. The appropriate action is an action with the highest probability of being rewarded by the environment. The interaction between the learning automaton and the random environment is shown in Fig. 3 [22].

Learning automata $(L A s)$ can be classified into two main families, fixed and variable structure learning automata [22]. Variable structure learning automata, which is used in this paper, is represented by sextuple $<\underline{\beta}, \underline{\phi}, \underline{\alpha}, \underline{\mathrm{P}}, \mathrm{G}, \mathrm{T}>$, where $\underline{\beta}$ is a set of input actions, $\underline{\phi}$ is a set of internal states, $\underline{\alpha}$ is a set of outputs, P denotes the state probability vector governing the choice of the state at each stage k, G is the output mapping, and $\mathrm{T}$ is a learning algorithm. The learning algorithm is a recurrence relation and is used to modify the probability vector.

It is evident that the crucial factor affecting the performance of the variable structure learning automata is the learning algorithm for updating the action probabilities. Let $\alpha_{i}$ be the action chosen at time $\mathrm{k}$ as a sample realization from distribution $\mathrm{p}(\mathrm{k})$. The linear reward-penalty algorithm $\left(\mathrm{L}_{\mathrm{RP}}\right)$ is one of the earliest schemes. In an $\mathrm{L}_{\mathrm{RP}}$ scheme the recurrence equation for updating probability vector $\mathrm{p}$ is defined by (4) for a favorable response $(\beta=1)$ and $(5)$ for an unfavorable response $(\beta=0)$.

$$
\begin{gathered}
p_{i}(k+1)=p_{i}(k)+a\left(1-p_{i}(k)\right) \\
p_{j}(k+1)=p_{j}(k)-a p_{j}(k), \forall j \neq i \\
p_{i}(k+1)=(1-b) p_{i}(k) \\
p_{j}(k+1)=\frac{b}{r-1}+(1-b) p_{j}(k), \forall j \neq i
\end{gathered}
$$

The parameters $a$ and $b$ represent reward and penalty parameters, respectively. The parameter $a(b)$ determines the amount of increases (decreases) of the action probabilities.

\section{Related Works}

In the review of the relevant literature, we specifically focus on studies addressing topology mismatch problems in unstructured peer-to-peer networks. Reported algorithms can be classified into three classes of algorithms (Fig. 4): algorithms in

Fig. 3 Learning automaton (LA)

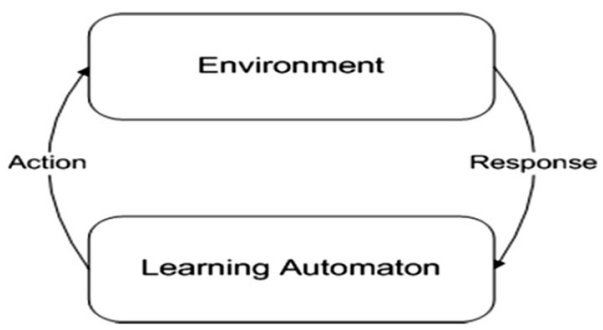




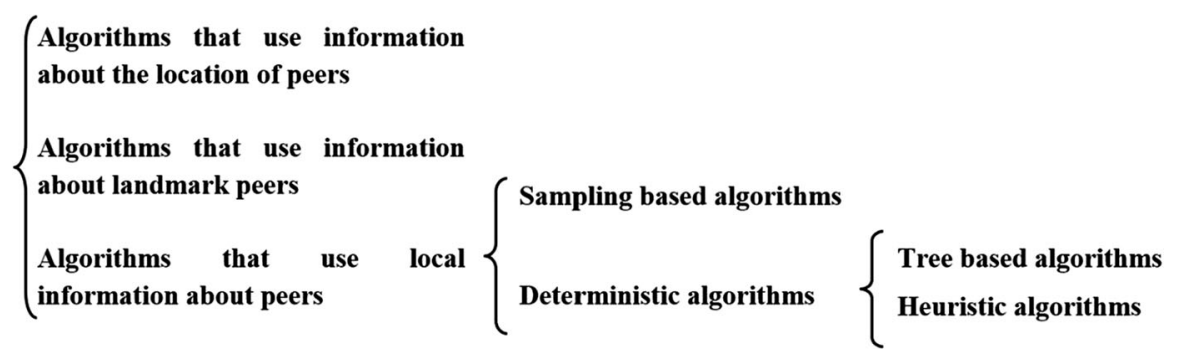

Fig. 4 A classification for topology matching algorithms

which each peer in the network uses some services such as GPS ${ }^{1}$ or Oracle to gather information about the location of peers [23-25], algorithms in which each peer in the network uses information about landmark peers [26-33], and algorithms in which each peer in the network uses local information about its neighbors $[6,7,9,11,12,16,17$, 34-39]. In the rest of this section, we briefly review these classes of algorithms.

Algorithms that use information about the location of peers: This type of algorithm uses several services to gather some information about the locations of peers. In [23, 24], the matching algorithm utilizes GPS to gather some information about the location of peers. In [25], an algorithm is suggested that uses oracle service to gather locality information about peers. Designing algorithms based on information about the location of peers creates new challenges such as the cost and availability of required services for peers.

Algorithms that use information about landmark peers: This type of algorithm uses some information about landmark peers. Landmark peers are specific peers in the network. In these algorithms such as those reported in [26-33, 40], the peers organize themselves into clusters so that the peers that get in within a given cluster are relatively close to each other in terms of communication delay. One of the drawbacks of these algorithms is that information about landmark peers may not always be available.

Algorithms that use local information about peers: These algorithms try to optimize the overlay structures using local information about peers. These algorithms can be further classified into two categories: sampling based algorithms and deterministic algorithms, which are described in the next two paragraphs.

In sampling based algorithms, such as those reported in $[9,36,37]$ some nonadaptive sampling methods are used to solve the topology mismatch problem. Since the status of peer-to-peer networks can change during the operation of the network, the non-adaptive sampling algorithms cannot be appropriate.

In deterministic algorithms, each peer uses a deterministic algorithm to find appropriate neighbors in order to solve the topology mismatch problem. Deterministic approaches can be further classified into two classes: tree based algorithms and heuristic algorithms. In tree based algorithms such as those reported in [16, 38, 39], some algorithms for finding minimum spanning trees and multicast trees are used to reconfigure the links of peers. A main drawback of tree based algorithms is that they not only need to tune their parameters with an adaptive mechanism, but they also need

\footnotetext{
${ }^{1}$ Global Positioning System.
} 
another algorithm to create multicast trees or minimum spanning trees. In heuristic algorithms, some local operators such as exchange operators use some local estimation to minimize mismatched links. Since the work presented in this paper is about heuristic algorithms, in the rest of this section we specially focus on heuristic algorithms.

In heuristic algorithms, some local operators such as exchange operators are used to decrease the mismatched paths of the overlay network. This type of algorithm consists of two parts: a local search operator to gather some information about neighbors of a peer and local operators to reconfigure the connections of that peer in order to minimize its mismatched paths. THANCS algorithm is a heuristic algorithm reported by Liu et al. in [7]. This algorithm adds links with low delay and removes those with high delay by checking peers within its neighborhood radius. THANCS is a well-known topology matching algorithm. In [11], a matching algorithm is presented by Rostami et al. This algorithm uses local information about peers to eliminate mismatched paths. Both the algorithm reported in [11] and THANCS are not able to improve their functionality considering the network conditions. In [35], the Location-aware Topology Matching (LTM) algorithm is reported by Liu et al. In this algorithm, the peers eliminate the redundant links of the overlay by measuring the delay of the overlay links. A main drawback of this algorithm is that a synchronization method must be implemented. In $[6,15]$, the PROP was designated by Qiu et al. This algorithm has two relevant policies: Generic $(P R O P-G)$ and Optimized $(P R O P-O)$. In $P R O P-G$, each peer searches its neighbors (within a particular distance) to select an appropriate peer and then exchanges all of its neighbors with the selected peer. In $P R O P-O$, a threshold is defined to determine the numbers of peers that can be used in each exchange operation. In $P R O P-O$, a peer searches its neighbors (within a particular distance) to select an appropriate peer and then exchanges an equal number of connections with that peer. $P R O P-O$ tries to preserve the number of connections of each peer during the execution of the matching algorithm. A main drawback of both $P R O P-O$ and $P R O P-G$ is that they have no self-adaptive mechanism to tune their parameters (such as parameter distance) of their local search algorithm. In $[12,17], X-B O T$ is proposed. $X$-BOT is an algorithm for topology optimization in unstructured peer-to-peer networks that can be used to solve the topology mismatch problem. This algorithm is able to bias an initial random overlay in order to optimize some efficiency criteria; for example, to reduce the delay of the overlay. This algorithm and $P R O P-O$ algorithm preserves the degree of nodes of the graph of the overlay network and the overlay connectivity. This algorithm has two main drawbacks. The first drawback is that this algorithm has several parameters which should be tuned by a designer. The second drawback is that the local search of this algorithm is not adaptive.

All of the heuristic algorithms reported in this section suffer from two problems. The first problem is that there is no adaptive mechanism for setting the neighborhood radius parameter that determines the scope of the local search of the matching algorithm. Finding an appropriate value for this parameter manually is a time consuming process and also error prone. Fixing this parameter is not also appropriate due to network dynamicity. The second problem is the lack of an adaptive mechanism for managing the execution of the local operator of the marching algorithm. To solve these problems a self-adaptive topology matching algorithm will be proposed in the next section. 


\section{The Proposed Algorithm}

In this section, a self-adaptive algorithm based on the learning automata and SSM for solving the topology mismatch problem will be proposed. In the proposed algorithm, each peer searches its neighbors to find an appropriate peer, and then uses the exchange operator to exchange its connections with that peer in order to reduce the number of mismatched paths of the overlay network. We define variable $r_{i}$ to save the neighborhood radius of peer $_{i}$. Each peer is equipped with a learning automaton. This learning automaton has two actions: "increase radius" and "decrease radius" and is responsible for adaptively tuning up the neighborhood radius. Figure 5 shows the interaction of the learning automaton $L A_{i}$ of peer $_{i}$ with its environment when $r_{i}=1$. The environment of the learning automaton $L A_{i}$ residing in peer peer $r_{i}$ is composed of the local environment of peer $_{i}$, and the global environment that consists of local environments of the peers, which are in the neighborhood of the peer ${ }_{i}$. In the proposed algorithm, for peer $_{i}$, a variable called $\mu_{i}$ is defined and can take a value of either "not moving" or "moving". This variable determines whether peer $_{i}$ can participate in the exchange operation or not. Function $\lambda_{i}$ takes information about the neighbors of peer $_{i}$ as the input and returns the portion of the neighbors of peer $r_{i}$ that cannot participate in the exchange operation to all neighbors of peer ${ }_{i}$. Variable $\mu_{i}$ and function $\lambda_{i}$ are defined to implement the process of changing neighbors of the agents of the SSM in the proposed algorithm and are responsible to manage the execution of the exchange operator. The formal definitions of all used variables and functions are given later. Algorithm 1 shows the high level structure of the proposed algorithm. This algorithm is briefly described in the next three paragraphs.

Once peer $_{i}$ joins the network, it first uses a neighborhood selection algorithm such as the neighborhood selection method of Gnutella for finding its neighbors and then executes the proposed algorithm. Before describing the phases of the proposed algorithm, the method used in Gnutella for the neighborhood selection is described in more detail as follows. In this method, peer $_{i}$ connects to global caches, which are called the Gnutella Web Caches. After connecting to the caches, peer $_{i}$ requests a list of peers. peer $_{i}$ tries to connect to the peers of the list, until it connects to a specific

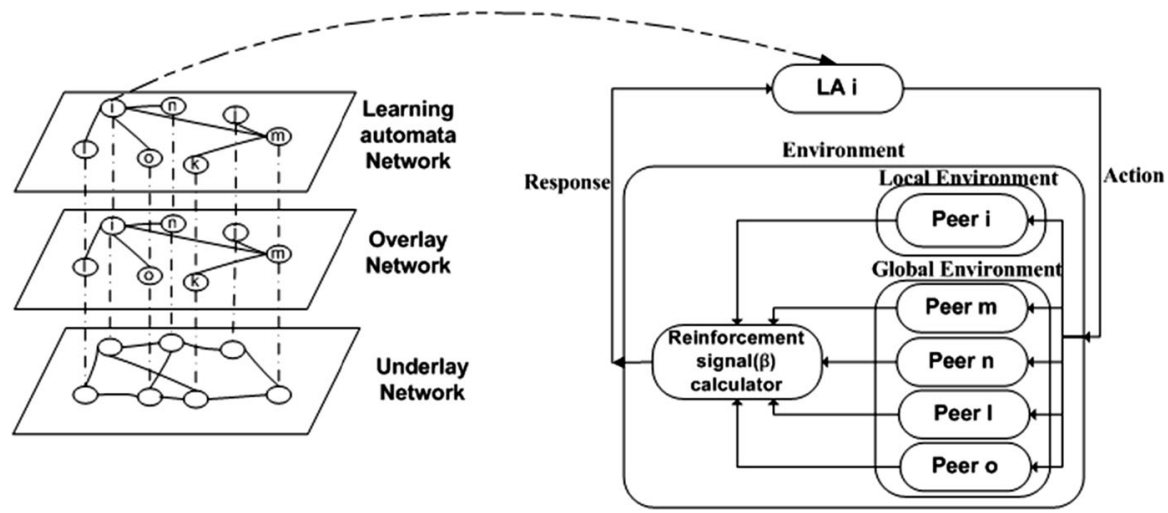

Fig. 5 Learning automaton for peer $_{i}$ and its environment 
number of peers [1]. Now we describe the phases of the proposed algorithm that uses information about delays among peers in order to solve the topology mismatch problem. The management algorithm consists of three phases: the local search phase, exchange phase, and maintenance phase. The phases of the proposed algorithm are summarized in Algorithm 1 and the procedure of the reinforcement signal calculator unit used by the learning automata is given in Algorithm 2. The phases of the proposed algorithm are briefly described in the next two paragraphs.

During the local search phase, peer $_{i}$ uses its learning automaton to determine the value of the neighborhood radius. If the value of the neighborhood radius is lower than or equal to 0 , the peer computes the value of the reinforcement signal (using the procedure reinforcement_signal_calculator given in Algorithm 2) otherwise, the peer activates all of its neighbors, exchange information about delays with its neighbors, and finds appropriate corresponding and candidate peers. Then peer $_{i}$ saves the found corresponding and candidate peers in its local database, computes the value of $\lambda_{i}$, finds the action selected by the majority of learning automata of neighboring peers, and finally computes the value of the reinforcement signal. peer $_{i}$ at the end of the local search phase asks its learning automaton to update its action probability vector and then goes to the exchange phase.

During the exchange phase, peer $_{i}$ first finds an appropriate corresponding peer, peer $_{j}$ from its local database to participate in the exchange operation. Then, peer $_{i}$ executes the exchange operator for exchanging its connections with peer $_{j}$, computes the value of $\lambda_{i}$, and sets the value of variable $\mu_{i}$. The peer sets the value of $\mu_{i}$ to "not moving" if the value of $\lambda_{i}$ is equal to 1 and set to "moving", otherwise. At the end of the exchange phase, if the value of variable $\mu_{i}$ is equal to "moving", peer $r_{i}$ goes to the local search phase, otherwise goes to maintenance phase. During the maintenance phase, which is an ever going process, peer $_{i}$ continually waits for one of the events of "changing connections by a neighboring peer" or "activation of matching algorithm by neighboring peer", which in that case, peer $_{i}$ restarts the matching algorithm. In the next paragraph, we describe how the concept of changing neighbors of agents in the SSM is used in the proposed algorithm.

The process of changing neighbors in the proposed algorithm plays the same role as the process of changing neighbors of agents in the SSM. In the proposed algorithm, similar to the $S S M$, peer $r_{i}$ changes its neighbors in order to increase the portion of neighboring peers $\left(\lambda_{i}\right)$ that do not have a mismatched path with peer $_{i}$. Since the value of the similarity function gives valuable information about the position of peer $_{i}$, it is used in the stopping condition and the reinforcement signal calculator unit of the proposed algorithm. The details about the similarity function are given later in the detailed descriptions of the proposed algorithm.

Before we present the proposed algorithm in more detail we need to define the following:

1. The neighborhood radius of peer $r_{i}$ of the algorithm is denoted by $r_{i}$. This parameter will be used to determine the broadcast scope of the control messages in the algorithm. $r_{i}$ is initially set to a given value.

2. The neighborhood set of peer $_{i}$ denoted by $N_{i}^{r_{i}}$ contains all peers residing in the neighborhood radius of peer $_{i}$, that is, $N_{i}^{r_{i}}=\left\{\right.$ peer $_{j} \in V \mid$ dist $\left(\right.$ peer $_{i}$, peer $\left.\left._{j}\right) \leq r_{i}\right\}$ 
Algorithm 1 peer_management_skeleton()

\section{Inputs:}

$m / /$ the initial value of the neighborhood radius of the peer

$t / /$ the threshold for the percentage of all neighbors of the peer that cannot participate in the exchange operation

\section{Notations:}

Let $L A$ determines the learning automaton of the peer

Let $\mu$ determines whether the peer can change its neighbors using exchange operation or not

Let $r$ determines the neighborhood radius of the peer

Let $\lambda$ determines the portion of the neighbors of the peer which cannot participate in the exchange operation

Let $\tau$ determines the portion of the neighbors of the peer which cannot participate in the exchange operation

Let $A$ determines the selected action of $L A$

Let $X$ determines the action selected by majority of learning automata of neighboring peers

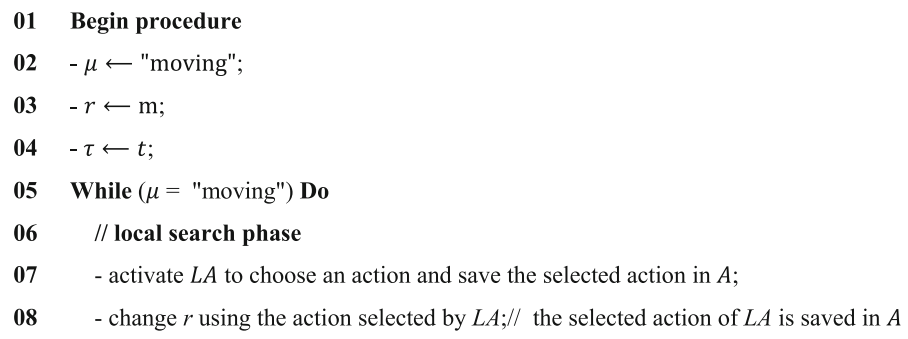

09 If $(r \leq 0)$ Then

$10-\beta \leftarrow$ reinforcement_signal_calculator $(r, \lambda, \tau, A, X)$;// the pseudo code of this procedure is given In Algorithm 2

11 Else

12 - activate all of neighbors of the peer determined by $r$;

13 - exchange information about delays with neighboring peers;

14 - find appropriate candidate and corresponding peers;

15 - save the identifiers of found corresponding and candidate peers into a local database in the peer;

- compute $\lambda$; //using information stored in the local database of the peer

17 - find the action selected by majority of learning automata of neighbors of the peer and save it in $X$;

$18-\beta \leftarrow$ reinforcement_signal_calculator $(r, \lambda, \tau, A, X)$; // the pseudo code of this procedure is given in Algorithm 2

\section{$19 \quad$ EndIf}

$20-L A$ updates its action probability vector using reinforcement signal $\beta$;

$21 / /$ exchange phase

22 - find an appropriate corresponding peer peer $_{j}$ from the local database of the peer;

23 - execute exchange operator with peer $_{j}$;

24 - compute $\lambda$; //using information stored in the local database of the peer and the information of new neighbors which are added during exchange operation If $(\lambda=1)$ Then 
where $\operatorname{dist}\left(\right.$ peer $_{i}$, peer $\left._{j}\right)$ is the length of the shortest path (with the minimum number of hops) between peer $_{i}$ and peer $_{j}$ in the overlay network. The immediate neighbors of peer $r_{i}$ is denoted by $N_{i}^{1}$.

3. The candidate peer set of peer $_{i}$ for peer $_{j}$ denoted by $C_{i j}$. This set contains some of the neighbors of peer $_{i}$, which change their connections from peer $_{i}$ to peer $_{j}$, during the exchange phase.

4. The corresponding peer set of peer $_{i}$ denoted by $M_{i}$. This set contains some of neighbors of peer $_{i}$ such as peer $_{j} \in N_{i}^{r_{i}}$ of whichpeer ${ }_{j}$ has a candidate peer set $C_{j i} \subseteq N_{j}^{1}$, and peer $_{i}$ has a candidate peer set $C_{i j} \subseteq N_{i}^{1}$ so that

$$
\left[\sum_{\text {peer }_{k} \in C_{i j}} d_{k i}+\sum_{\text {peer } C_{l} \in C_{j i}} d_{l j}\right]>\left[\sum_{\text {peer }} \in C_{j i} d_{l i}+\sum_{\text {peer }_{k} \in C_{j i}} d_{k j}\right] .
$$

In other words, $M_{i}$ contains some of the neighbors of peer ${ }_{i}$ that can participate in the exchange operation to decrease the number of mismatched paths of the overlay network.

5. $\lambda_{i}=\frac{\left|N_{i}^{1}\right|-\left|M_{i}\right|}{\left|N_{i}^{1}\right|}$ is the portion of neighboring peers that do not have a mismatched path with peer $_{i}$ (the portion of the neighbors of peer $_{i}$ which cannot participate in the exchange operation). The similarity function $\lambda_{i}$ used in the proposed algorithm is designated based on the fact that when a peer has no mismatched paths with its neighbors $\left(\left|M_{i}\right|=0\right)$, the peer should stop executing the matching algorithm. The value of $\lambda_{i}$ increases during the exchange operation and reaches 1 when peer $_{i}$ has no mismatch path $\left(\left|M_{i}\right|=0\right)$. The value of the similarity function gives valuable information about the position of the peer and is used for computing the reinforcement signals of the learning automata.

6. Candidate table of peer $_{i}$ denoted by $C T_{i}$ saves information about the corresponding peers and the candidate peers of peer $_{i}$. This information is required by the algorithm to compute the reinforcement signal for the learning automaton of peer $_{i}$.

7. There are five types of control messages: ExchangeRequest, ExchangeReady, UpdateState, ReleaseRequest and ReleaseReply that are used in the proposed 


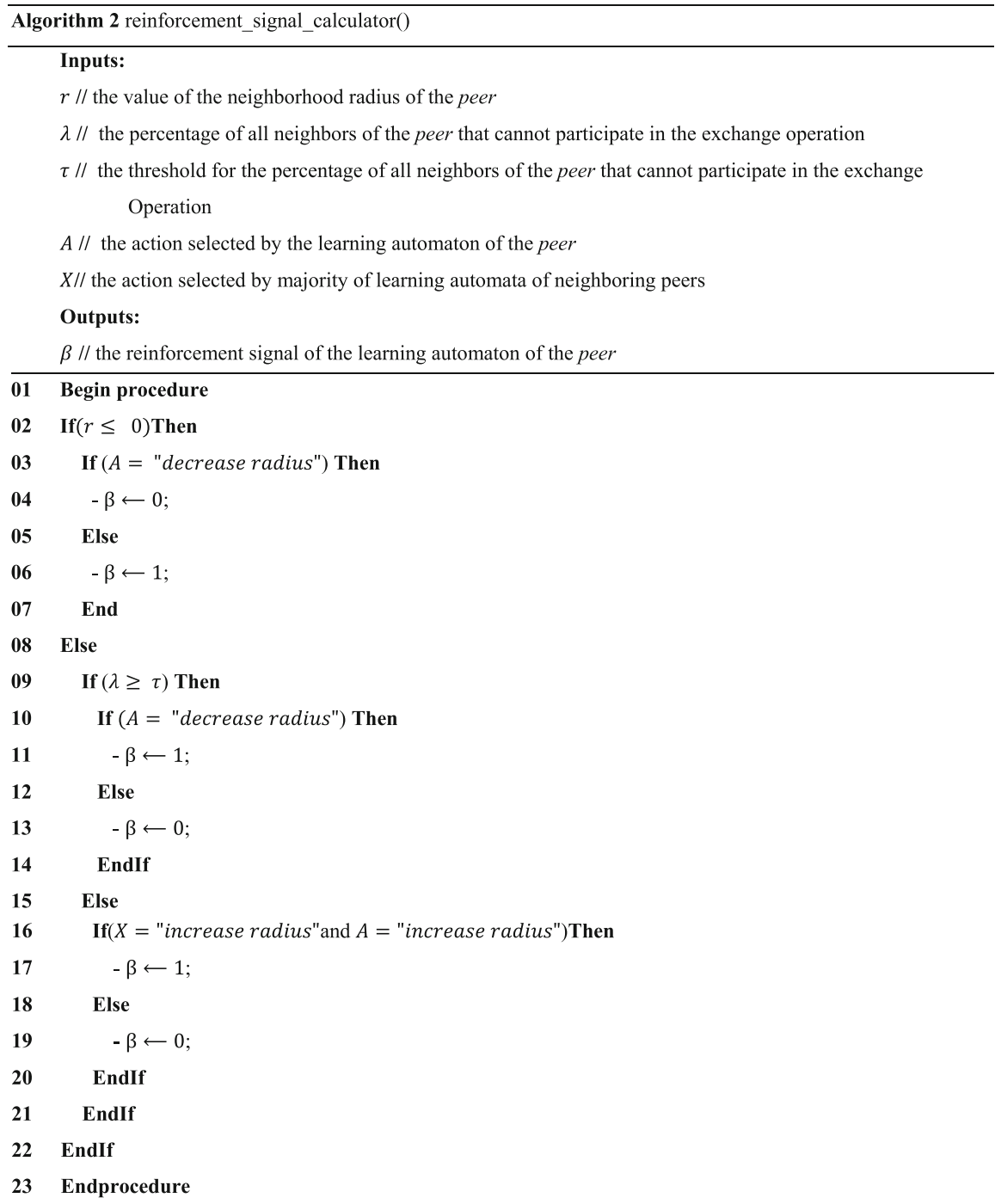

algorithm. Table 1 shows the fields of these messages. Field Type contains the type of the message. Field TTL contains the "time to live value" for the message to ensure that the message dies out. The neighborhood radius is used to determine the TTL value. Sender and Receiver fields contain the sender or receiver identifier (such as the IP addresses). Field Action contains either the "Increase radius" or "Decrease radius". Field Candidate-Peers contains the identifiers for candidate peers, and field Delay contains the delays for candidate peers.

Now we describe the three phases of the process LA_Match. Algorithm 3 shows the pseudo code for process $L A \_$Match performed by peer $_{i}$. Once peer $_{i}$ joins the network it uses the neighborhood selection method of Gnutella to find its neighbors, 
Table 1 The format of the used messages

\begin{tabular}{ll}
\hline Message name & The fields of the message \\
\hline ExchangeRequest & Type, Sender, TTL, Candidate-Peers \\
ExchangeReady & Type, Sender, Receiver, TTL, Action, Candidate-Peers, Delay \\
ReleaseRequest & Type, Sender, Receiver, TTL, Candidate-Peers \\
ReleaseReply & Type, Sender, Receiver, TTL \\
UpdateState & Type, Sender,TTL \\
\hline
\end{tabular}

broadcast UpdateState messages to all its neighbors, and then executes the proposed management algorithm. The detailed descriptions of the proposed algorithm are given in the remainder of this section.

\subsection{Local Search Phase}

The local search phase starts with the initialization phase and ends with the candidate selection phase as described below.

Initialization phase: During the initialization phase performed by peer learning automaton $L A_{i}$ randomly selects one of its actions "Increase radius" or "Decrease radius" according to its action probability vector. The new neighborhood radius $r_{i}$ for peer $_{i}$ is then computed accordingly. If $r_{i}$ is greater than or equal to one, then peer $_{i}$ generates an ExchangeRequest message that contains its immediate neighbors as candidate peers and broadcasts it to all of its neighbors and then goes to the candidate selection phase. If $r_{i}$ is less than one, then peer $r_{i}$ computes the reinforcement signal of the $L A_{i}$ using the reinforcement_signal_calculator procedure given in Algorithm 2. After computing the reinforcement signal, $L A_{i}$ updates its probability vector and then peer $_{i}$ calls the exchange procedure to start the exchange phase. The pseudo code of the exchange procedure is given in Algorithm 4.

Candidate selection phase: In this phase, peer ${ }_{i}$ waits for a certain duration (SEARCH_DURATION) to receive an ExchangeRequest message or ExchangeReply message from its neighbors. peer $_{i}$ upon receiving an ExchangeRequest message from peer $_{j}$, which contains the candidate peers, and finds the delays between itself and the candidate peers. peer $r_{i}$ then sends an ExchangeReply message to peer $_{j}$. This message contains the immediate neighbors of peer $_{i}$, the delays between peer $_{i}$ and the immediate neighbors, the delays between peer $_{i}$ and the candidate peers, and the action selected by $L A_{i}$ during the Initialization phase. peer ${ }_{i}$ then restarts the candidate selection phase.

peer $_{i}$ upon receiving an ExchangeReply message from peer $_{j}$, which contains the candidate peers and the delays of the candidate peers, finds the delay between itself and the candidate peers. peer $r_{i}$ then uses all of the gathered information about the delays of links to determine whether peer $_{j}$ can be a corresponding peer or not. If peer $_{i}$ can select some of its neighbors as $C_{i j}$ (the candidate peer set for peer $_{j}$ ) and select some of the candidate peers as $C_{j i}$ (the candidate peer set for peer ${ }_{i}$ ) so that $\left|C_{j i}\right|=\left|C_{i j}\right|$ and $\left[\sum_{\text {peer }} \in C_{i j} d_{k i}+\sum_{\text {peer } r_{l} \in C_{j i}} d_{l j}\right]>\left[\sum_{\text {peer } r_{l} \in C_{j i}} d_{l i}+\sum_{\text {peer }} \in C_{j i} d_{k j}\right]$ then peer $_{j}$ is 
a corresponding peer. If peer $r_{j}$ is a corresponding peer, then peer $_{i}$ inserts peer $_{j}$ as its corresponding peer and the selected peers $\left(C_{i j}\right.$ and $\left.C_{j i}\right)$ as the candidate peer sets into its candidate table. peer $r_{i}$ then restarts the candidate selection phase. If peer $_{i}$ does not receive any messages during the specified period of the SEARCH_DURA$T I O N$, then it computes the reinforcement signal for learning automaton $L A_{i}$. After computing the reinforcement signal, $L A_{i}$ updates its probability vector, and then peer $_{i}$ goes to the exchange phase by calling exchange procedure.

Note that for selecting candidate peer sets, peer $_{i}$ generates all possible candidate peer sets and then compares the generated sets with each other in order to find appropriate candidate peer sets. That is, peer $r_{i}$ selects two candidate peer sets $\left(C_{i j}\right.$ and $C_{j i}$ ) in such a way that the difference between the sum of the end-to-end delays of the network before and after the exchange operation becomes the maximum.

\subsection{Exchange Phase}

During this phase, peer $_{i}$ for each corresponding peer $_{j}$ given in its candidate table, generates a ReleaseRequest message containing all the candidate peers of peer $_{j}$ and then sends it to the corresponding peer $r_{j}$. After sending the ReleaseRequest messages to all the corresponding peers, peer $_{i}$ waits for certain duration (EXCHANGE_DURATION) to receive the ReleaseReply messages or ReleaseRequest messages from its neighbors.

peer $_{i}$, upon receiving a ReleaseRequest message that contains candidate peers from peer $_{j}$, finds $C_{i j}$ and $C_{j i}$, creates new links to the candidate peers that are available in $C_{j i}$, removes the old links to the candidate peers that are available in $C_{i j}$, sends a ReleaseReply message to peer ${ }_{j}$, and returns to process the $L A \_$Match that is called the exchange phase. peer $r_{i}$ sends ReleaseReply messages to peer $_{j}$ via two paths: a path that uses the candidate peers that are connected to the corresponding peer peer ${ }_{j}$ and a path that exists between peer $_{i}$ and peer $_{j}$ to ensure that ReleaseReply messages reach peer $_{j}$.

peer $_{i}$, upon receiving a RelaseReply from peer ${ }_{j}$, goes to its candidate table and finds those candidate peers whose corresponding peer is peer ${ }_{j}$. Then it finds $C_{i j}$ and $C_{j i}$, creates new links to the candidate peers that are available in $C_{j i}$, removes the old links to the candidate peers that are available in $C_{i j}$, sends an UpdatedState message to its neighbors, and returns to the process $L A \_$Match that is called the exchange phase. If peer $_{i}$ does not receive any messages during the specified period of EXCHANGE DURATION then it returns to process $L A \_$Match that is called the exchange phase.

When peer $_{i}$ returns to the $L A \_$Match process, if the portion of its neighbor that cannot participate in the exchange operation is equal to one then its variable $\mu_{i}$ is set to "not moving" and it goes to the maintenance phase. peer ${ }_{i}$ restarts the local search phase when its variable $\mu_{i}$ is equal to "moving".

\subsection{Maintenance Phase}

During this phase, peer $_{i}$ waits until some event occurs. peer $_{i}$ restarts the matching algorithm if it receives UpdateState or ExchangeRequest messages and restarts the maintenance phase, otherwise. 


\section{Algorithm 3 LA_Match}

\section{Inputs:}

$m / /$ the initial value of the neighborhood radius

$t / /$ the threshold for the percentage of all neighbors of the peer that cannot participate in the exchange operation

\section{Notations:}

Let $r$ determines the neighborhood radius of the peer

Let $\mu$ determines whether the peer can participate in exchange operation or not

Let $\lambda$ determines the portion of the neighbors of the peer which cannot participatein the exchange operation

Let $C T$ determines the candidate table of the peer

Let $L A$ determines the learning automaton of the peer

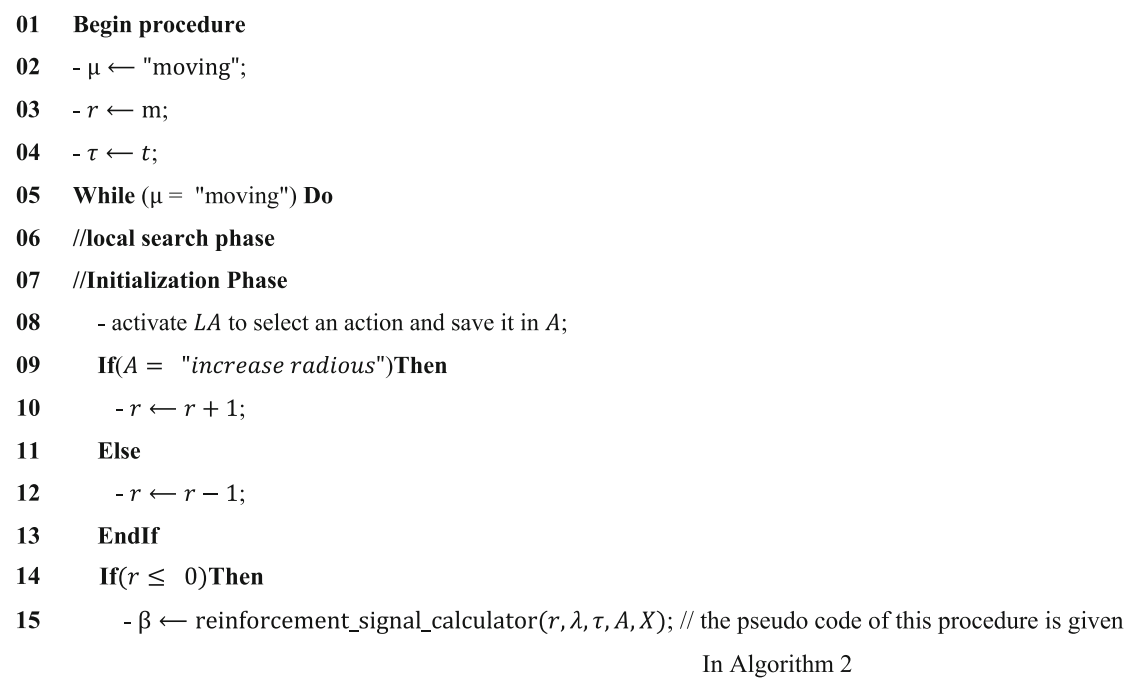

Else

- Broadcast ExchangeRequest message containing the immediate neighbors to all the neighbors

determined by $r$;

18 // Candidate selection phase

$19-$ - isSelectionCompleted $\leftarrow$ False;

$20 \quad$ Repeat

- wait for a certain duration SEARCH_DURATION for ExchangeRequest or ExchangeReply message;

If(no message has been received during SEARCH_DURATION) Then

- isSelectionCompleted $\leftarrow$ True;

Else

If(ExchangeRequest message has been received from another peer $_{j}$ ) Then 
to

candidate

peer $_{j} ; / / A$ is the action chosen by $L A$ during Initialization phase

\section{EndIf}

If (an ExchangeReply message has been received from peer $_{j}$ ) Then

- find delays to candidate peers;//candidate peers are reported by ExchangeReply message

- select candidate peer sets;//select candidate peer sets using all delays which are found and

reported by ExchangeReply message

If(the candidate peer sets are not empty) Then

- insert peer $_{j}$ as the corresponding peer and the selected candidate peer sets of peer ${ }_{j}$ in table $C T$;

\section{EndIf}

$38 \quad$ Until (isSelectionCompleted)

39 - compute $\lambda$; //using $C T$

40 - find the action selected by majority of learning automata of neighboring peers and save it in $X$;

$/ / X$ is found using information provided by ExchangeReply messages

$41-\beta \leftarrow$ reinforcement_signal_calculator $(r, \lambda, \tau, A, X)$; // the pseudo code of this procedure is given in Algorithm 2

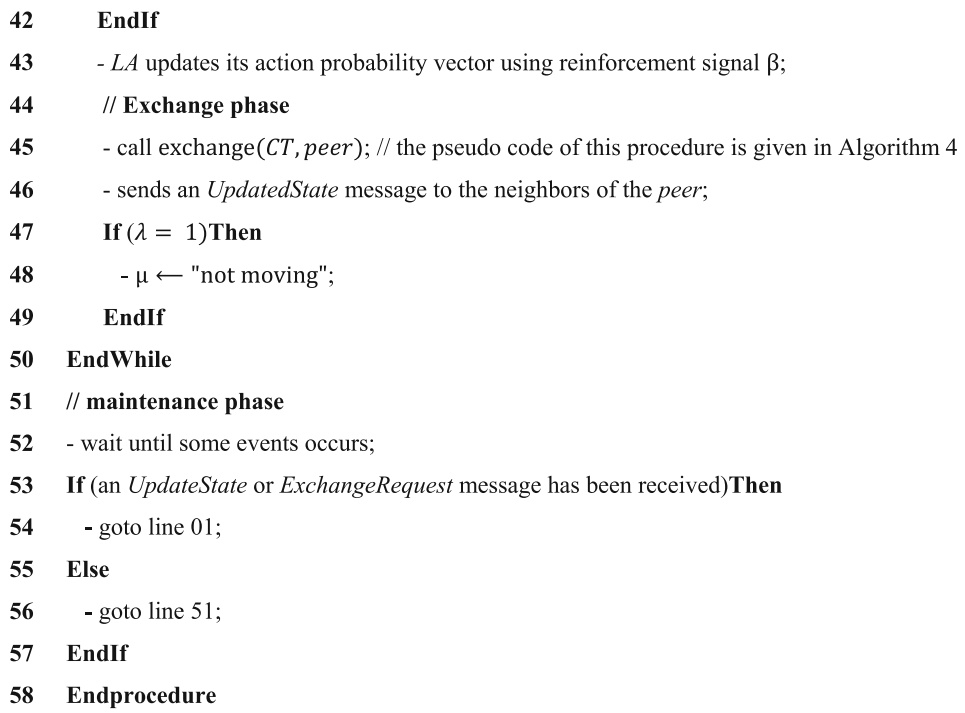




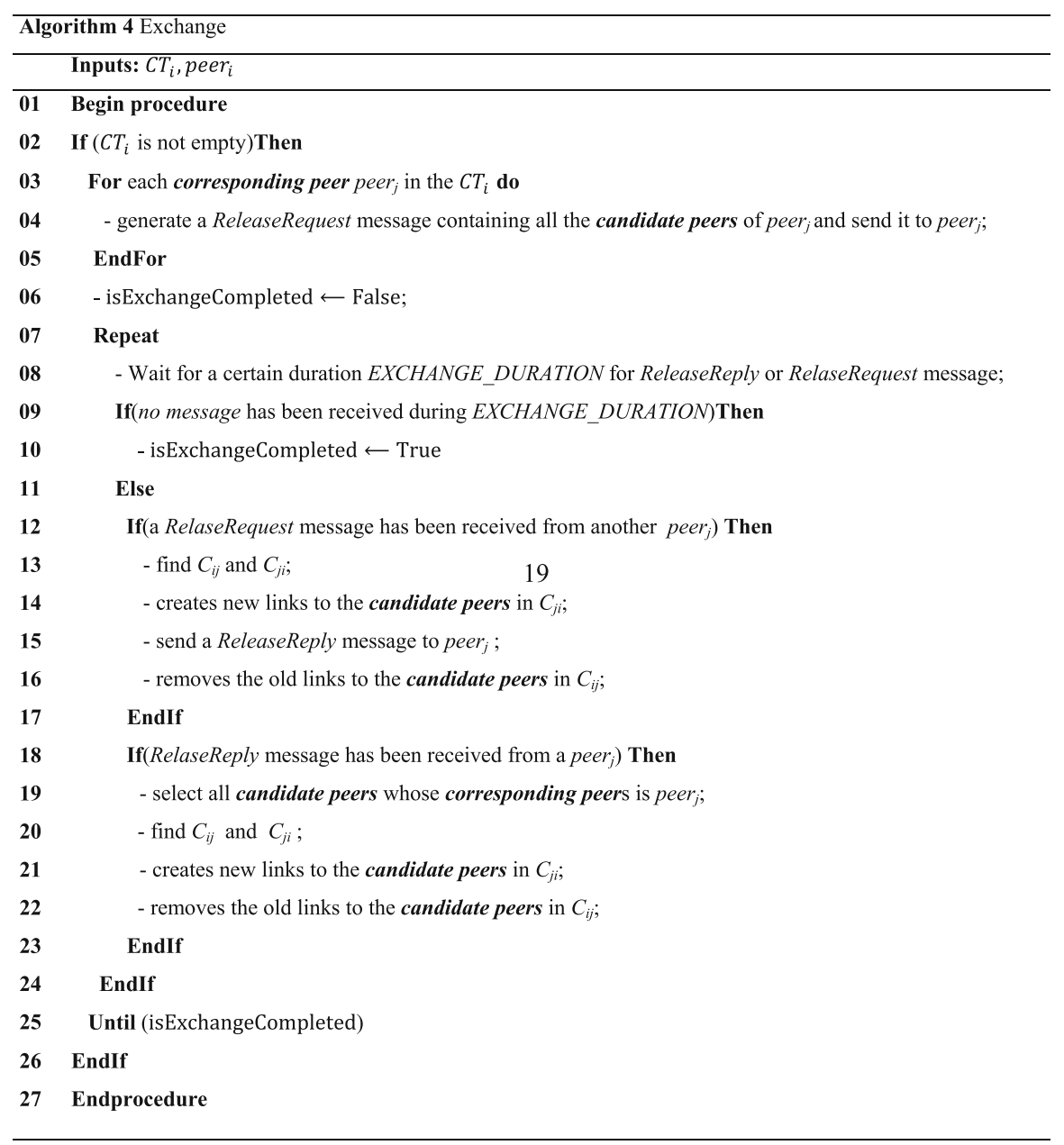

\section{Experimental Results}

All simulations have been implemented using OverSim that is an overlay network simulation framework [41, 42]. OverSim supports different kinds of underlay network models: Simple, SingleHost, INET, and ReaSE [43]. Simple model is the most scalable one and the ReaSE model is able to generate different types of underlay networks. We used the ReaSE model to generate router-level topologies [44]. Experiments reported in this section are conducted on three different underlying networks: Topology.1 as an example of the Simple model, and Topology. 2 and Topology. 3 as examples of the ReaSE model, as given in Table 2. For the overlay network management algorithm, Gnutella [45] and GIA [2] are used. Since Gnutella is used to test several matching algorithms reported in [6, 7, 9, 36$39,46]$ and it is a peer-to-peer network with an open and well-documented protocol 
specification, we use it as the default overlay network management algorithm in this section. We used the Gnutella dataset of [47] to generate the initial overlay topology. This data set contains a graph where its nodes, edges, and diameter are $10,876,39,994$ and 9, respectively. In an experiment, GIA is used as the overlay network management algorithm. GIA is fully implemented in Oversim. The Random churn model [41] and Pareto churn model [41] are also used to model the process of joining and leaving peers in the network.

To evaluate the performance of the proposed algorithm called LA_Match, it is compared with three algorithms: PROP-O [6, 15], X-BOT [12, 17], and THANCS [7]. It should be noted that, the proposed algorithm, like algorithms PROP-O,X$B O T$, and THANCS (to which the proposed algorithm is compared) uses only the delays of links for solving the topology matching problem. Other algorithms, such as those reported in [23-33, 40] use other information, such as geographical positions and information about landmark peers, which may not always available. Both $P R O P-O$ and $X-B O T$ algorithms, like the proposed algorithm use a management operator (called the exchange operator) to exchange connections among the peers to maintain the number of connections of each peer during the execution of the matching algorithm. Other algorithms, such as those reported in [9, $11,16,35-39$ ] change the number of connections of peers that are not appropriate for some peer-to peer networks. The $P R O P-O$ algorithm has a parameter called $n h o p s$ that plays the same role as parameter $m$ in the proposed algorithm. The reason of selecting THANCS is that it is a well-known algorithm for topology matching in unstructured peer-to-peer networks.

The proposed algorithm has four parameters: $m, t, S E A R C H \_D U R A T I O N$, and EXCHANGE_DURATION. Two parameters SEARCH_DURATION and EXCHAN$G E \_D U R A T I O N$ must be set to large values in order to provide enough time for the peers of the network to communicate with their neighbors. For the experiments, SEARCH_DURATION is set to $5 s$, and EXCHANGE_DURATION is set to $5 s$. To test the impact of parameters $m$ and $t$ on the performance of the algorithm and also to show the learning capability of the proposed algorithm, several experiments were conducted as reported in the remaining part of this section. For all experiments, each peer is equipped with a variable structure learning automaton of type $L_{R P}$ with a

Table 2 Underlay topologies

\begin{tabular}{ll}
\hline $\begin{array}{l}\text { Underlay } \\
\text { topology }\end{array}$ & Descriptions \\
\hline Topology.1 & $\begin{array}{l}\text { In this underlay topology, peers are placed on a N-Dimensional Euclidean space and } \\
\text { the Internet latencies are based on CAIDA/Skitter [48, 49] data }\end{array}$ \\
Topology.2 & $\begin{array}{l}\text { Consists of } 10 \text { autonomous systems, and about } 1087 \text { router-level nodes. This topology } \\
\text { contains few and populated group }\end{array}$ \\
Topology.3 & $\begin{array}{l}\text { Consists of } 50 \text { autonomous systems, and about } 217 \text { router-level nodes. This topology } \\
\text { contains many low populated groups in which the distance between the groups is far } \\
\text { greater than the distance between the peers in each group }\end{array}$ \\
\hline
\end{tabular}


reward parameter $a=0.25$ and a penalty parameter $b=0.25$. The other parameters of the PROP-O algorithm are set as follows: $M I N_{-} V A R=0, M A X_{-} I N I T_{-}-$ TRIAL $=10, \quad m=3$, INIT_TIMER $=10 \mathrm{~min}$, MAX_TIMER $=1000 \mathrm{~min}$. The parameters of the $X-B O T$ algorithm are set as follows: $k=6, P B O=10 \mathrm{~s}$, $\pi=2$, and $\mu=3$. In the THANCS algorithm each peer flood control messages periodically (every $5 \mathrm{~s}$ ) to gather required information about its neighbors. Experiments 1, 2, and 3 are conducted to find the most appropriate parameters for the proposed algorithm to be used in the later experiments. Results reported are averages over ten different runs.

The algorithms are compared with respect to four metrics: overlay communication delay, mismatched paths, overlay reconfiguration overhead, and control message overhead. These metrics are briefly explained below:

- Overlay communication delay $(O C D)$ is the sum of end-to-end delays of links in the overlay network (using 3).

- Number of Mismatched Paths $(M P)$ is the number of all mismatched paths of the overlay network. A mismatched path is a path in an overlay network that in its corresponding underlay path, a particular position has been visited more than once.

- Overlay reconfiguration overhead $(O R O)$ is the number of peers that are reconfigured by a matching algorithm. This metric implicitly shows the changes made by the local operators (such as exchange operators) of the matching algorithms.

- Control message overhead (CMO) is the number of extra control messages generated for the purpose of reconfiguration of an overlay network.

\subsection{Experiment 1}

This experiment is conducted to study the effect of parameter $t$ on the performance of the proposed algorithm when parameter $m$ is set to 1. For this study, the proposed algorithm is tested for three initial values for parameter $t=0.3,0.6$ and 0.9 , and Topology. 1 is used as the underlay network. The results are compared with respect to the four above mentioned criteria. According to the results of this experiment, which are shown in Fig. 6, one may conclude the following:

- In terms of $O C D$ and $M P$, the proposed algorithm (with $t=0.6$ or $t=0.9$ ) performs better than the proposed algorithm with $t=0.3$. This is because a low value for parameter $t$ leads to low sensitivity of the proposed algorithm of a peer to the information about other peers of the network. It should be noted that in each peer, parameter $t$ implicitly determines whether or not the information about neighboring peers should be considered in the reinforcement signal calculator unit of the proposed algorithm. Since the knowledge about the neighbors of the peers gives valuable information about the state of the network, a low value for parameter $t$ lead to conduct the learning automata of the peers to converge to inappropriate actions which lead to a low accuracy of the proposed 
(a)

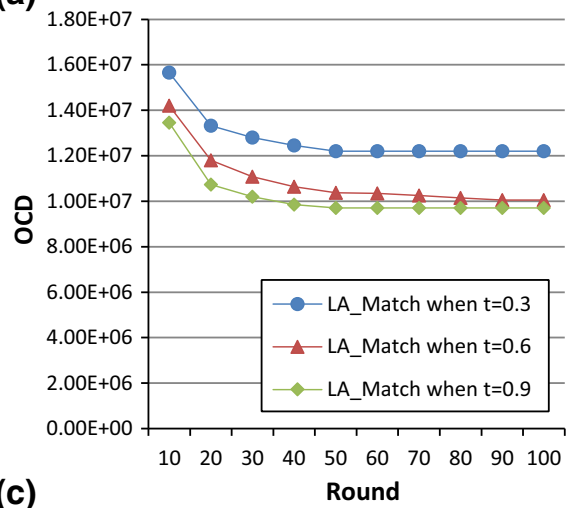

(c)

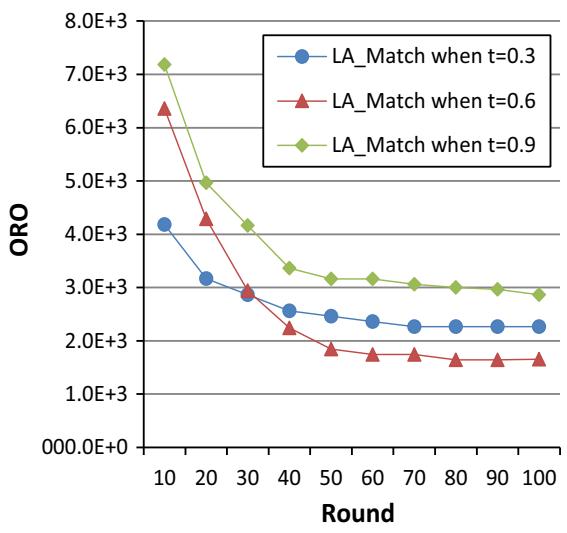

(b)

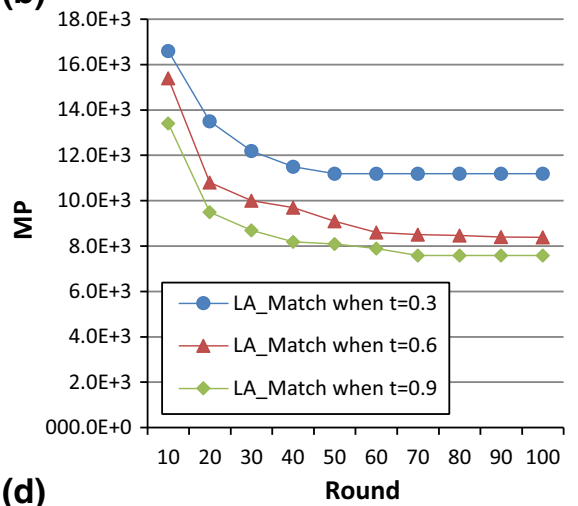

(d)

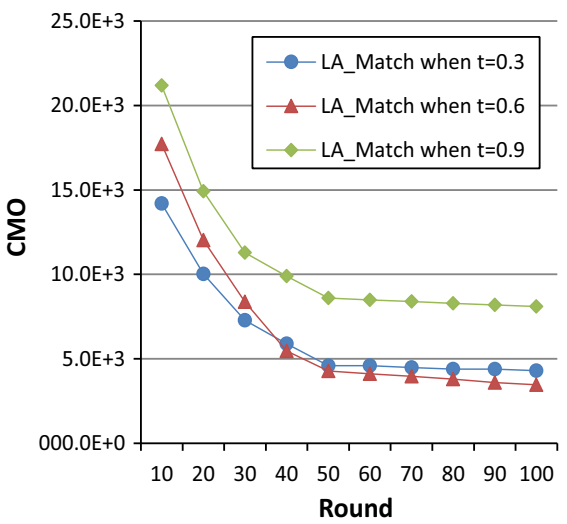

Fig. 6 The effect of parameter $t$ on the performance of $L A \_M a t c h$ with respect to a $O C D, \mathbf{b} M P, \mathbf{c} O R O$, d $C M O$

algorithm (with respect to a high $O C D$ and $M P$ ) and high overhead caused by the algorithm (with respect to a high $C M O$ and $O R O$ ).

- In terms of $O R O$ and $C M O$, the proposed algorithm with $t=0.6$ performs better than the proposed algorithm with $t=0.3$ or $t=0.9$ except for the early rounds of the simulation.

- Increasing the value of parameter $t$ leads to a decrease in $O C D$ and $M P$, but increases $O R O$ and $C M O$ in the early rounds of the simulation. This means that by increasing the value of parameter $t$, we can find an appropriate overlay with a low $O C D$ (overlay communication delay) and a low $M P$ (number of mismatched paths). However, we will have a high overhead (with respect to $C M O$ and $O R O$ ) in the early rounds of the simulation.

- From round 40 to round 100 , the proposed algorithm (when $t=0.9$ ) does not perform well with respect to $C M O$ and $O R O$ but does perform well with respect to $O C D$ and $M P$. In other words, for a high value for parameter $t$, when the overlay topology is changed to an appropriate overlay (with respect to a low $O C D$ and $M P$ ), the overhead of the proposed algorithm remains high (with 
respect to a high $C M O$ and $O R O$ ). This is because the learning automata of the peers do not decrease the parameters neighborhood radius of peers even after changing the overlay topology to an appropriate one. A high value for parameter $t$ leads to high sensitivity of the proposed algorithm to information about peers of the network. Since the knowledge about the neighbors of some peers of the network may not reflect the whole state of the network, a high value for parameter $t$ may conduct the learning automata of some peers of the network to converge to inappropriate actions that lead to set the neighborhood radius of the peers to large values. It is obvious that large values for the parameter neighborhood radius lead to generating many control messages and performing many unnecessary changes in the network that leads to a high $C M O$ and $O R O$.

\subsection{Experiment 2}

This experiment is conducted to show the impact of the parameter $m$ (neighborhood radius) on the performance of the proposed algorithm when the parameter $t$ is set to 0.6. For this purpose, the proposed algorithm is tested for three initial values for parameter $m=1,2$, and 3 and Topology. 1 is used as underlay topology. The results are compared with respect to the four above mentioned criteria. According to the results of this experiment that are shown in Fig. 7, one may conclude the following:

- Increasing the value of parameter $m$ leads to improving the performance of the proposed algorithm in terms of $O C D$ and $M P$. This is because a large radius gives each peer of the overlay more appropriate corresponding and candidate peers during a local search. Appropriate corresponding and candidate peers can participate in an exchange operation to decrease more mismatched paths that lead to low $O C D$ and $M P$.

- Increasing the value of parameter $m$ results in high $C M O$ and $O R O$ at early rounds of the simulation. Note that the parameter $m$ affects the scope of the local search in the proposed algorithm. In a peer, parameter $m$ determines a set of peers so that the information about them should be used by the reinforcement calculator unit of the matching algorithm in that peer. Increasing the value of parameter $m$ results in gathering more information about the current state of the network in an early round of the simulation. Gathering more information results in generating more control messages (with respect to a high CMO) and more peers can be reconfigured (with respect to a high $O R O$ ). Note that, gathering more information about the network enables each peer to find appropriate links to other peers in order to solve the topology mismatch problem which leads to a low $O C D$ and $M P$.

- It can be noted from the results that as the time passes the performance of the proposed algorithmin in terms of $C M O$ and $O R O$ improves. This is because, after finding appropriate neighbors by a peer, the peer is able to decrease its neighborhood radius using its own learning automata. Decreasing the neighborhood radius leads to a decrease in the scope of the local search, and decreasing the scope of the local search leads to a low CMO and ORO. 
(a)

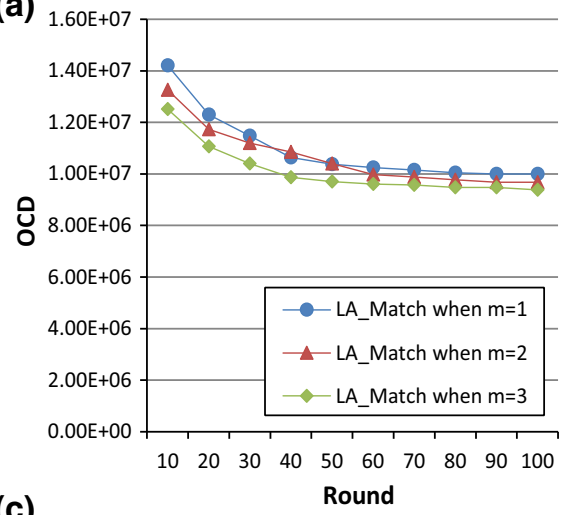

(c)

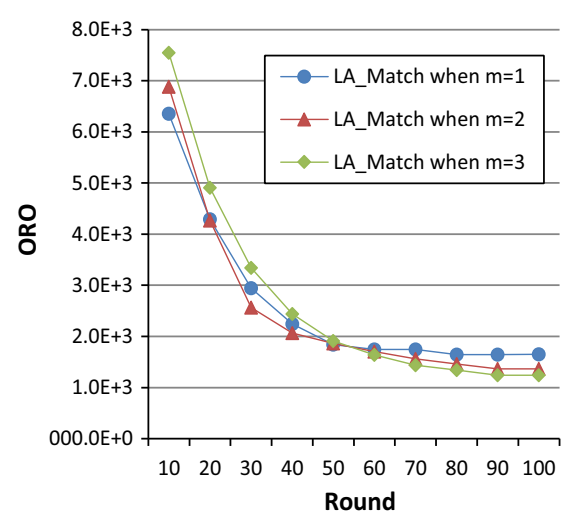

(b)

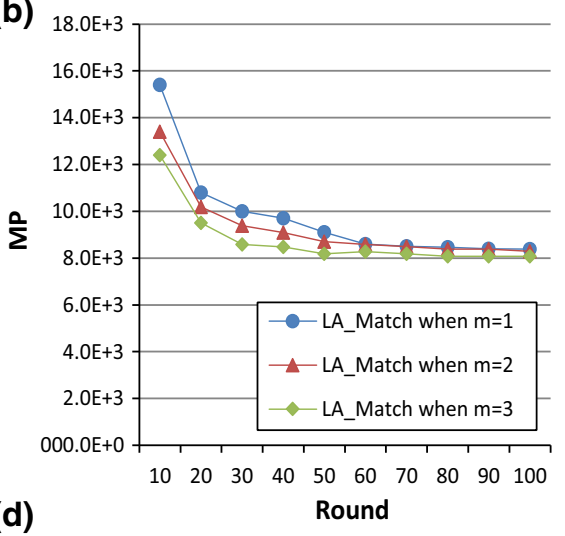

(d)

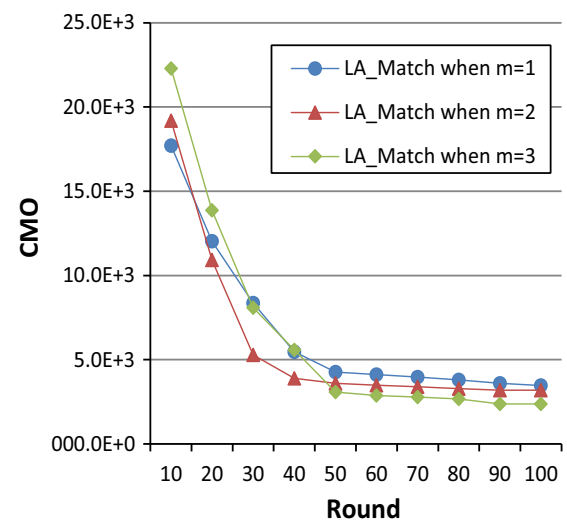

Fig. 7 The effect of parameter $m$ on the performance of $L A \_$Match with respect to a $O C D, \mathbf{b} M P$, c $O R O, \mathbf{d} C M O$

\subsection{Experiment 3}

This experiment is conducted to compare the proposed algorithm with (1) the proposed algorithm in which the learning automaton residing in each peer is deactivated ( $L A \_$Match_D algorithm); this is the proposed algorithm without leaning capability; (2) the proposed algorithm in which each learning automaton is replaced with a learning automaton that has three actions, "increase radius", "don't change radius", and "decrease radius" (LA_Match_M algorithm); and (3) the proposed algorithm in which each learning automaton is replaced with a pure chance automaton(LA_Match_P algorithm). In a pure chance automaton the actions of automaton are always selected with equal probabilities [50]. For this experiment, parameter $m$ is set to 1 and parameter $t$ is set to 0.6 and Topology. 1 is used as the underlay topology. The results of this experiment are given in Fig. 8. From the result we may conclude the following: 


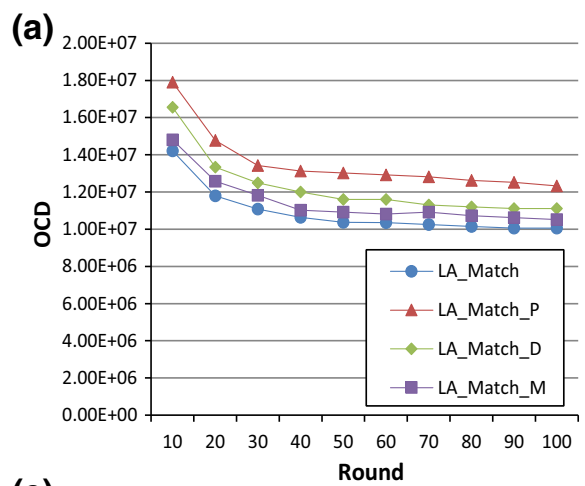

(c)

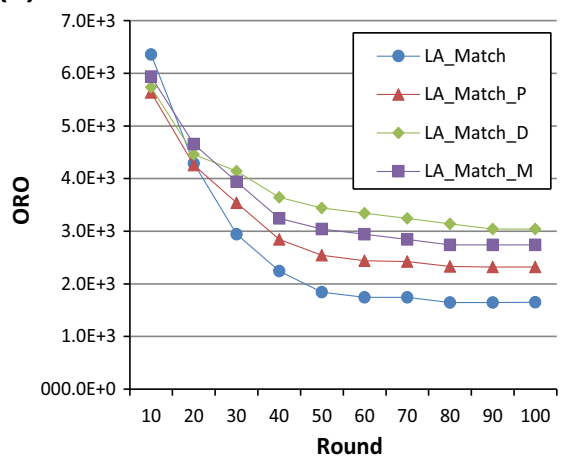

(b)

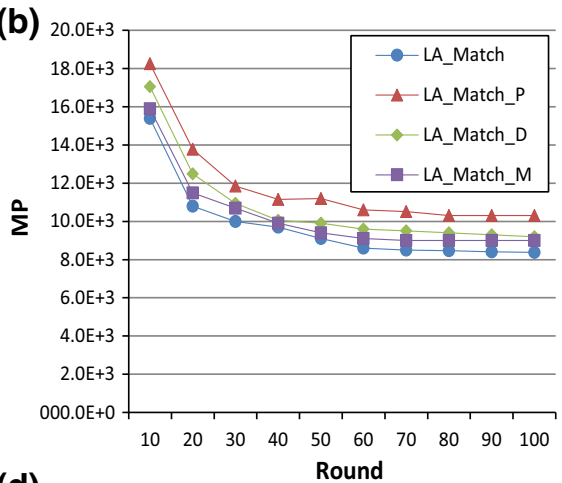

(d)

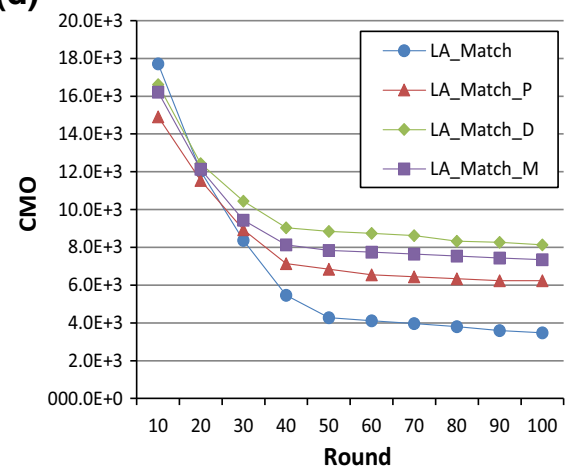

Fig. 8 Comparison of different versions of the proposed algorithm with respect to a $O C D, \mathbf{b} M P, \mathbf{c} O R O$, d $C M O$

- In terms of $O C D, M P, C M O$, and $O R O$, the LA_Match and LA_Match_M algorithms perform better than other algorithms (LA_Match_D and $L A \_$Match_P) except for the early periods of the operation of the network. This is because of the fact that in $L A \_$Match and $L A \_M a t c h \_M$ algorithms (because of their learning capability) improve their performance by updating their action probabilities according to the reinforcement signal received from the network. This reinforcement signal, which is computed based on some information about the peers and their neighbors reflects the current state of the network, and it is used to improve the behavior of the learning automata in finding the appropriate neighborhood radius. Finding the appropriate neighborhood radius leads to finding better candidate peers and corresponding peers resulting in fewer numbers of mismatched paths and also a lower $O C D, C M O$ and $O R O$.

- $\quad L A \_M a t c h$ performs better than $L A \_M a t c h \_M$ in terms of $C M O$ and $O R O$. This is because in $L A \_M a t c h \_M$, the learning automaton of a peer may converge to "don't change radius", which leads to fixing the neighborhood radius for the peer. When the neighborhood radius of a peer is fixed and the peer cannot find an appropriate position within its neighborhood, then many unnecessary control 
messages will be generated by the peer (leading to a higher CMO) or unnecessary changes may occur in connections of the peer with other peers (leading to an increase $O R O$ ).

- From the results of this experiment we may conclude that the learning automata with two actions ("increase radius" and "decrease radius") can be sufficient for this problem. This is because the stopping condition used in the LA_Match can conduct the process of matching without using additional action for the learning automata. In the LA_Match, the matching algorithm tries to change its neighborhood radius when it has a mismatched path with its neighbors. In a peer $r_{i}$, the value of $\lambda_{i}$ is equal to a portion of the neighboring peers that haven't the mismatched path to the peer $_{i}$, and it is used in the stopping condition of the algorithm. When peer $_{i}$ has no mismatched path with its neighbors, the matching algorithm is stopped and peer $_{i}$ does not need to execute the matching algorithm so it isn't forced to learn a specific neighborhood radius. Therefore, a learning automaton with two actions ("increase radius" and "decrease radius") can be sufficient for this problem.

(a)

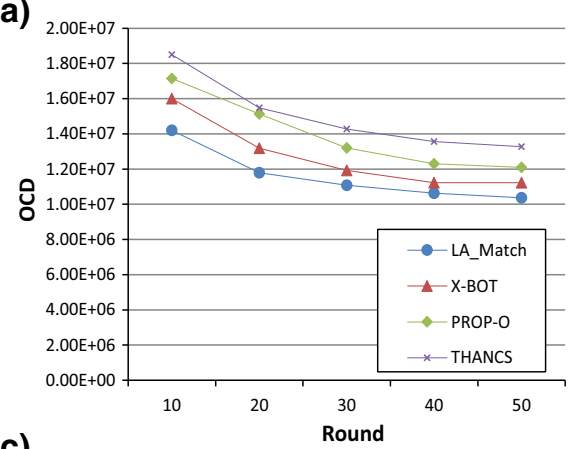

(c)

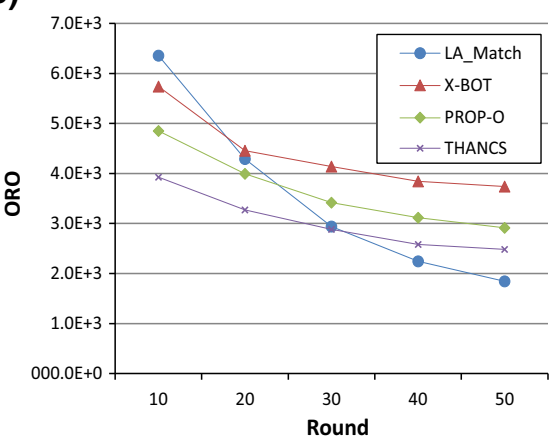

(b)

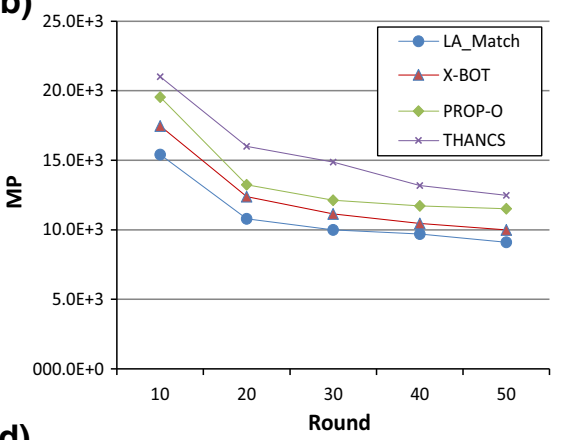

(d)

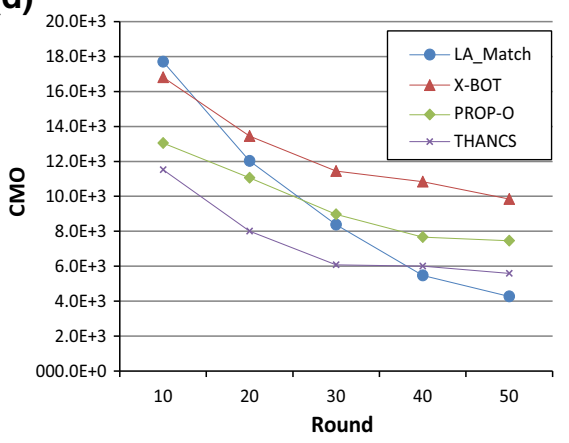

Fig. 9 Comparison of $L A \_M a t c h$ with existing matching algorithms with respect to a a $O C D, \mathbf{b} M P$, c $O R O, \mathbf{d} C M O$ 


\subsection{Experiment 4}

In this experiment, we compare the proposed algorithm with $X-B O T, P R O P-O$, and THANCS algorithms with respect to $O C D, M P, C M O$, and $O R O$. In this experiment, parameters $m$ and $t$ of the proposed algorithm are set to 1 and 0.6 respectively, and Topology. 1 is used as the underlay topology. According to the results of this experiment which are shown in Fig. 9, we may conclude that in term of $O C D, M P$, $C M O$, and $O R O$, the proposed algorithm performs better than $X-B O T, P R O P-O$, and THANCS algorithms except for the early periods of the operation of the network. This is because of the fact that in the proposed algorithm, each peer utilizes a learning automaton for parameter adaptation (parameter neighborhood radius) and a mechanism inspired from the SSM for adaptive execution of the exchange operator that improves its functionality as the network operation proceeds. Adaptation of parameter $m$ helps each peer to find appropriate corresponding and candidate peers to be used in the exchange operation leading to a lower $O C D$ and $M P$. Adaptive execution of the exchange operator also helps each peer to manage the exchange operators in such a way that unnecessary exchange operations are avoided and lead to a lower $O R O$ and $C M O$. In contrast to $X-B O T$, PROP-O, and THANCS algorithms, which do not utilize adaptive mechanisms to improve their functionality, the proposed algorithm (by utilizing an adaptive mechanism) is able to

(a)

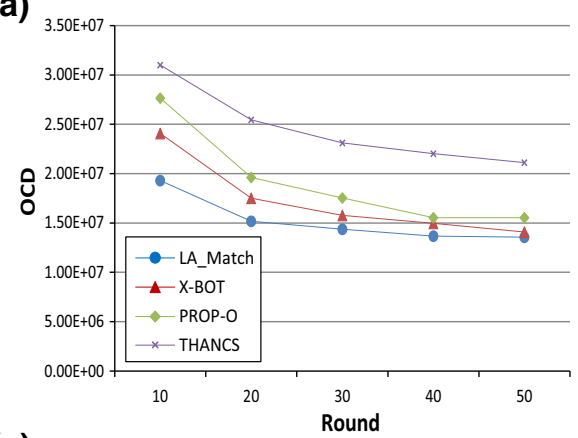

(c)

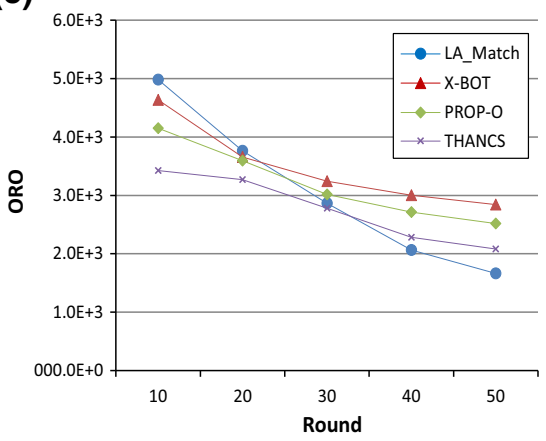

(b)

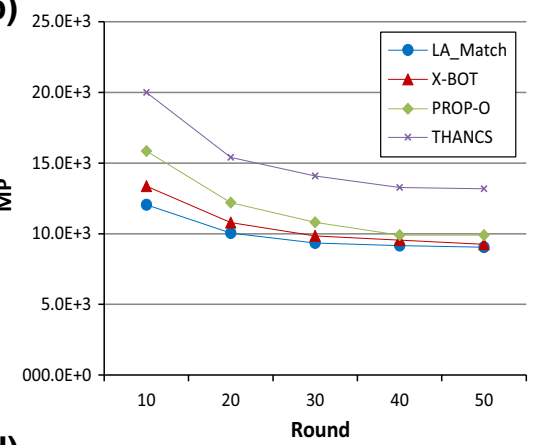

(d)

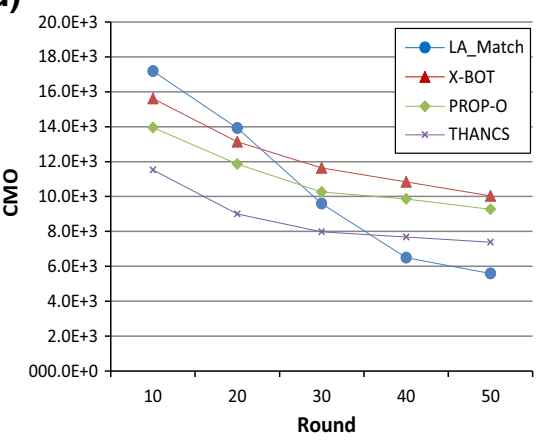

Fig. 10 Comparison of $L A \_M a t c h$ with existing matching algorithms with respect to a $O C D, \mathbf{b} M P$, c $O R O, \mathbf{d} C M O$ when $G I A$ is used 
improve the quality of the overlay (with respect to a low $O C D$ and $M P$ ) and decrease its overhead (with respect to a low $O R O$ and $C M O$ ).

\subsection{Experiment 5}

In this experiment, we study the performance of the proposed algorithm when $m=1$ and $t=0.6$ on an overlay that utilizes GIA to manage the overlay topology. In this experiment, Topology. 1 is used in the underlay network. The results are compared with the results obtained for $X-B O T, P R O P-O$, and THANCS algorithms in terms of $O C D, M P, C M O$, and $O R O$. In this experiment, each peer uses GIA to find its neighbors and then executes the matching algorithm ( $L A \_M a t c h, P R O P-O$, $X-B O T$, and THANCS) to modify its connections to its neighbors in order to solve the topology mismatch problem. From the results given in Fig. 10, we can say that the proposed algorithm performs better than other algorithms in terms of $O C D, M P$, $C M O$, and $O R O$ except for the early rounds of the simulation. This means that, the proposed algorithm can be efficient even for GIA. This is because the proposed algorithm is equipped with a self-adaptive mechanism and its design is not dependent on a specific overlay network, and therefore, it can be useful for different types of overlay networks.

(a)

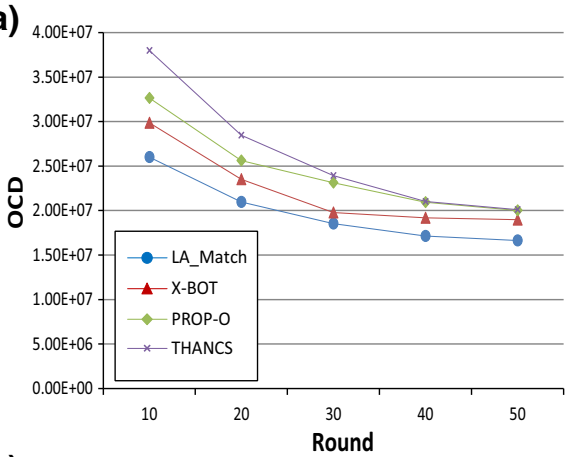

(c)

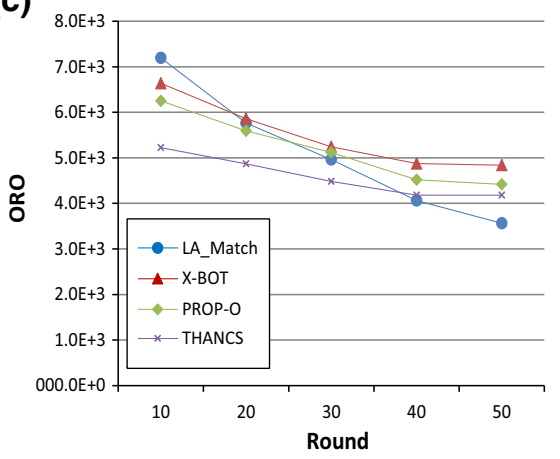

(b)

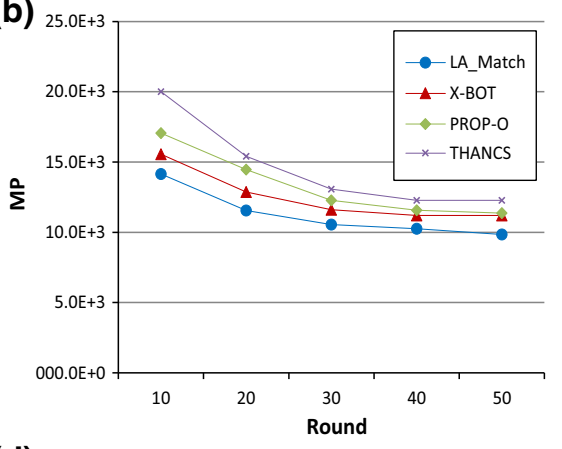

(d)

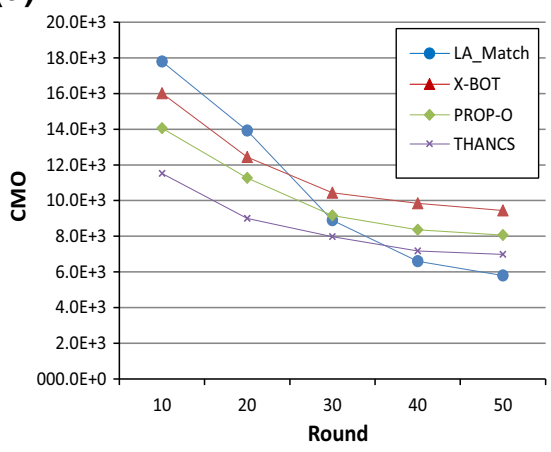

Fig. 11 Comparison of $L A \_M a t c h$ with existing matching algorithms with respect to a $O C D, \mathbf{b} M P$, c $O R O$, d $C M O$ when Topology. 2 is used 


\subsection{Experiment 6}

In this experiment, we compare the proposed algorithm with $P R O P-O, X-B O T$, and THANCS algorithms when topologies Topology. 2 and Topology.3 have been used. Both Topology.2 and Topology.3 belong to the class of router level topologies [44]. Topology. 2 contains few populated groups, but Topology.3 contains many low populated groups in which the distance between the groups is far greater than the distance between the peers in each group. In other words, in Topology.3 the probability that two peers in the overlay network belong to different autonomous systems is high. Parameters $m$ and $t$ of the proposed algorithm are set to 1 and 0.6 , respectively. The results are compared with respect to four criteria $O C D, M P, C M O$, and $O R O$. Figure 11 give the results of this experiment for Topology. 2 and Fig. 12 for Topology.3. From the results one may conclude the following:

- In terms of $O C D$ and $M P$, the proposed algorithm outperforms $X-B O T, P R O P$ $O$, and THANCS algorithms when Topology. 3 is used. This is because (1) for Topology. 3 the probability that any pair of peers in the overlay network selected by the exchange operator be far apart from each other is high and (2) the proposed algorithm is able to find more appropriate candidate peers due to the

(a)

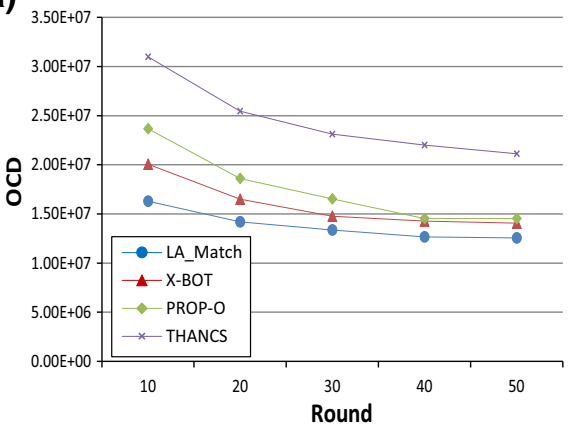

(c)

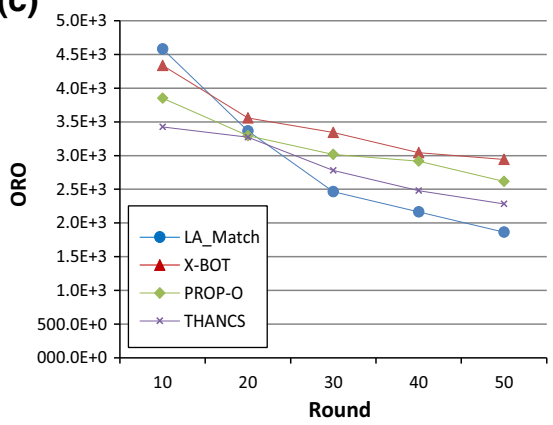

(b)

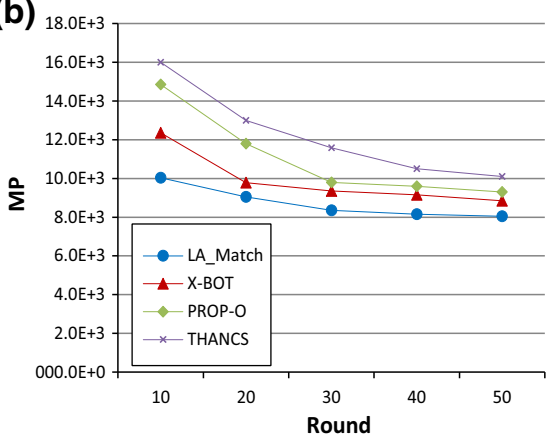

(d)

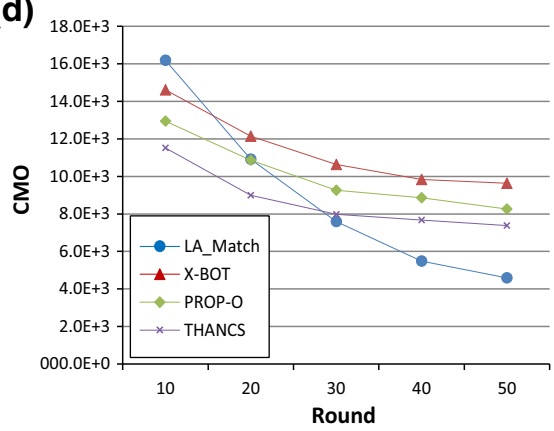

Fig. 12 Comparison of $L A \_M a t c h$ with existing matching algorithms with respect to a $O C D, \mathbf{b} M P$, c $O R O$, d $C M O$ when Topology. 3 is used 
adaptation of the parameter radius, and therefore, the exchange operator of the proposed algorithm can significantly reduce both $O C D$ and $M P$.

- In terms of $O C D$ and $M P$, the proposed algorithm performs better than $X-B O T$, PROP-O, and THANCS algorithms when Topology. 2 is used. Since Topology. 2 has large and populated groups, for other matching algorithms that use local search with fixed neighborhood radii take some number of rounds until a peer finds an appropriate candidate to be exchanged with. This leads to a higher $O C D$ and $M P$.

Table 3 Churn model setting

\begin{tabular}{lll}
\hline Churn model type & Churn model name & Churn model parameter \\
\hline Random churn model [41] & Random churn 1 & joining_probability $=0.8$ \\
Random churn model [41] & leaving _probability $=0.2$ \\
Pareto Churn model [41] & Random churn 2 & joining _probability $=0.7$ \\
& & leaving _probability $=0.3$ \\
& Pareto Churn 1 & Lifetime mean: $90 \mathrm{~s}$ \\
\hline
\end{tabular}

(a)

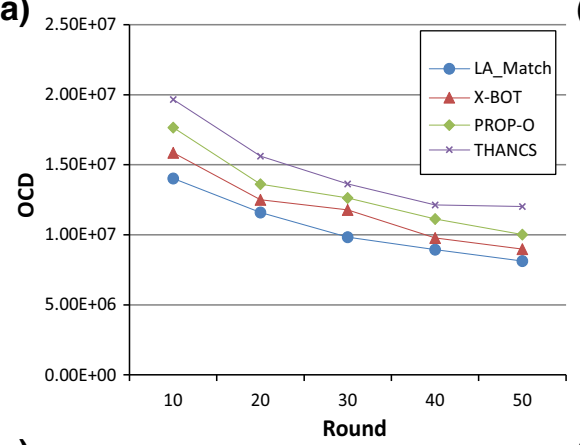

(c)

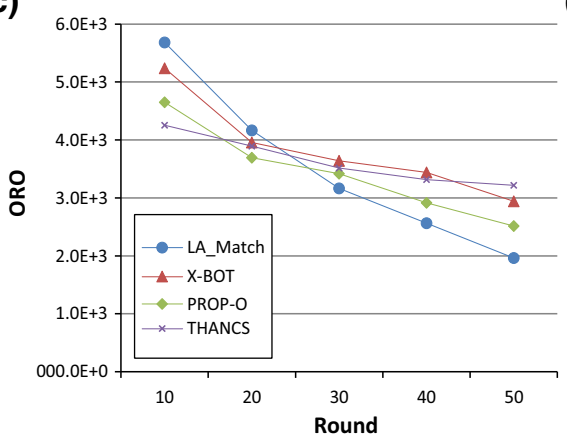

(b)

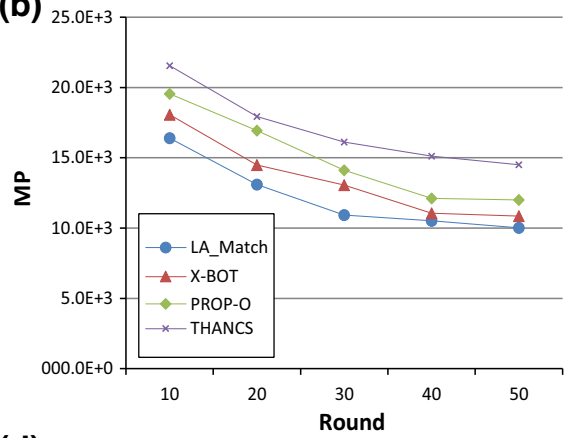

(d)

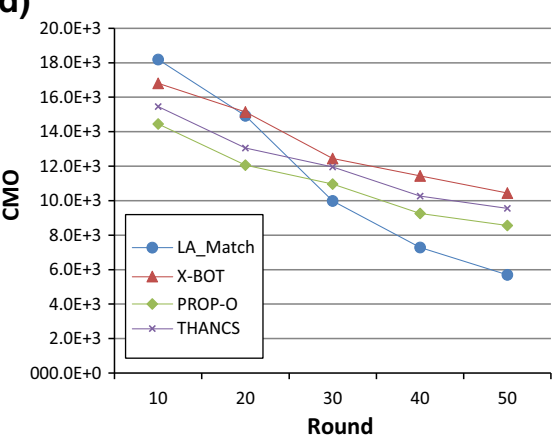

Fig. 13 Comparison of $L A \_M a t c h$ with existing matching algorithms with respect to a $O C D, \mathbf{b} M P$, c $O R O$, d $C M O$ when Random churn 1 is used 

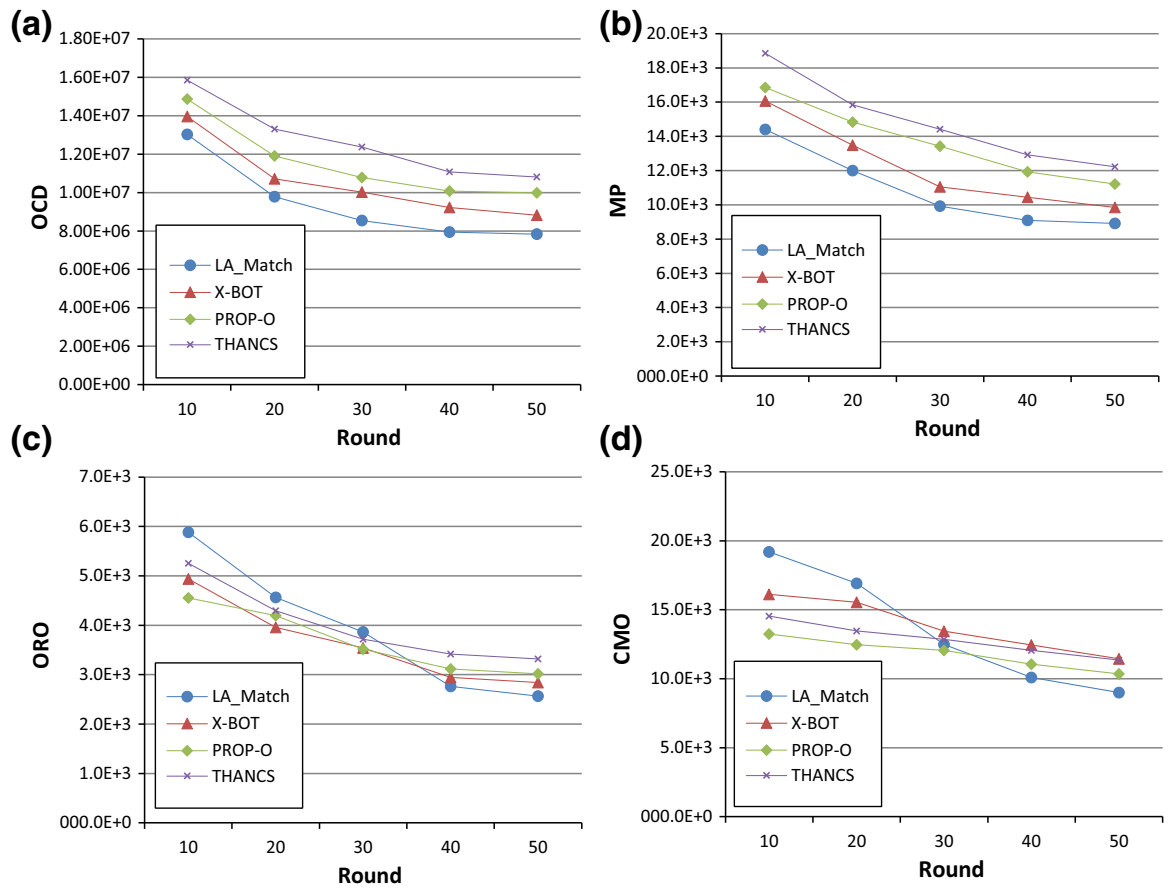

(d)

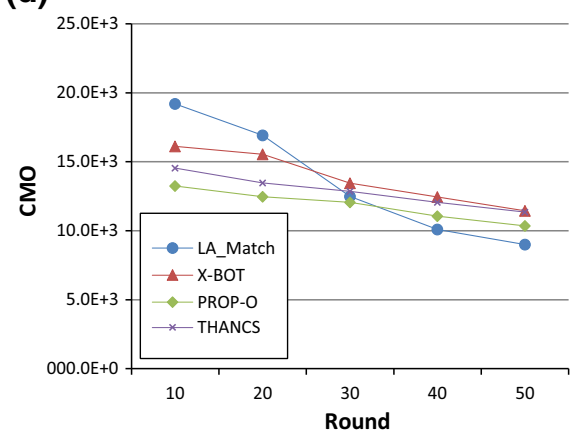

Fig. 14 Comparison of $L A \_$Match with existing matching algorithms with respect to a $O C D, \mathbf{b} M P$, c $O R O, \mathbf{d} C M O$ when Random churn 2 is used

- From the results of this experiment, we can conclude that the proposed algorithm can be efficient even for router level topologies. This is because the proposed algorithm uses some information about delays of links, and delays of the link reflect information about the structure of the underlay network. Therefore, the overlay network utilizing the proposed algorithm is able to change its configuration considering information about the underlay network.

\subsection{Experiment 7}

This experiment is conducted to study the effect of different churn models on the performance of the proposed algorithm when $m=1$ and $t=0.6$. The churn models used are the Random churn model [41] and Pareto churn model [41] according to Table 3. In Table 3, the probabilities of joining and leaving the peers for two Random churn models and the lifetime mean and the dead time mean for one Pareto churn model are given. The results obtained for the proposed algorithm are compared with the results for the $X-B O T, P R O P-O$, and THANCS algorithms with respect to $O C D, M P, O R O$, and $C M O$. Figures 13,14 and 15 give the results of this experiment.

From the result we may conclude the following: 
(a)

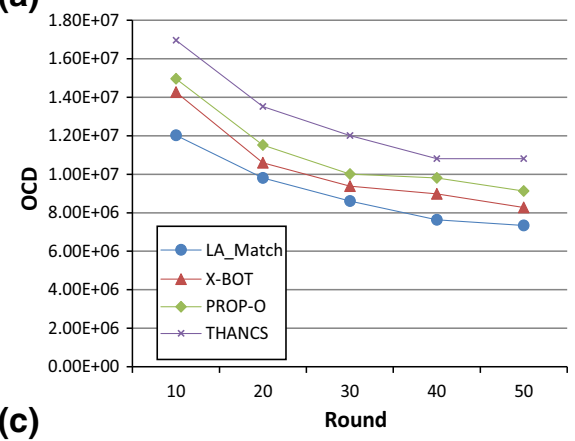

(c)

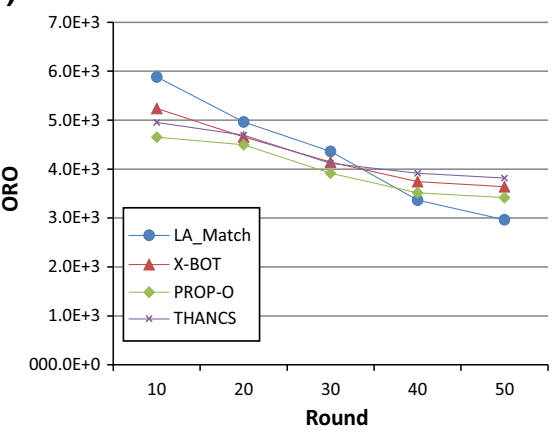

(b)
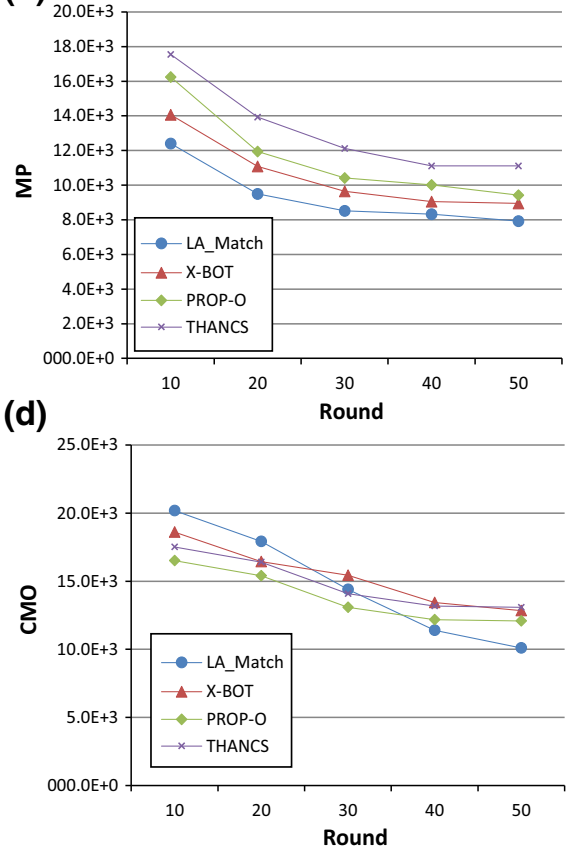

Fig. 15 Comparison of $L A \_M a t c h$ with existing matching algorithms with respect to a $O C D, \mathbf{b} M P$, c $O R O, \mathbf{d} C M O$ when Pareto Churn 1 is used

- In terms of overlay communication delay $(O C D)$ and number of mismatched paths $(M P)$ the proposed algorithm performs better than PROP-O, X-BOT, and THANCS algorithms for all churn models. This is because of the fact that the proposed algorithm tunes up its neighborhood radius in a self-adaptive manner that leads to finding better candidate peers and therefore, results in a fewer number of mismathed paths and also a lower $O C D$.

- In terms of control message overhead $(C M O)$ and overlay reconfiguration overhead $(O R O)$, the proposed algorithm performs worse than other algorithms at early rounds of the simulation for all the churn models. This is because in the proposed algorithm, each peer cooperates with its neighbors to improve the decisions of its management unit during the operation of the network. The information about the joining and leaving peers will be propagated among peers because of the cooperation among peers. The information propagation among peers increases the amount of control messages of the algorithm (with respect to a high $C M O$ ) and motivates the management units of more peers to consider the changes that were made in the network and which lead to an increase in the changes of the configuration of the peers (with respect to a high $O R O$ ). It should be noted that $X-B O T, P R O P-O$, and THANC have no mechanism to mitigate the negative effects of the churn. 


\section{Conclusion}

In this paper, an algorithm based on learning automata and the SSM to solve the topology mismatch problem was proposed. The proposed algorithm uses learning automata to improve its local search operation and the SSM to improve its connection establishment process. Improving the local search operation causes more appropriate corresponding and candidate peers to be found and used by the exchange operation leading to decreasing the number of mismatched paths and also decreasing the delays of the paths of the overlay network. Improving the connection establishment process helps each peer to better manage the exchange operators that prevent performing unnecessary exchange operations that lead to decreasing the number of reconfigured peers and the number of control messages of the matching algorithm. To evaluate the proposed algorithm, several computer experiments have been conducted using OverSim. Experimental results showed that the proposed algorithm can compete with some of the existing matching algorithms including $X$ $B O T, P R O P-O$, and THANCS algorithms in terms of overlay communication delay and the number of mismatched paths.

\section{References}

1. Kwok, Y.K.: Peer-to-Peer Computing: Applications, Architecture, Protocols, and Challenges. CRC Press, Boca Raton (2011)

2. Chawathe, Y., Ratnasamy, S., Breslau, L., Lanham, N., Shenker, S.: Making gnutella-like p2p systems scalable. In: Proceedings of the Conference on Applications, Technologies, Architectures, and Protocols for Computer Communications, pp. 407-418. ACM, Karlsruhe (2003)

3. Clarke, I., Sandberg, O., Wiley, B., Hong, T.: Freenet: a distributed anonymous information storage and retrieval system. In: Designing Privacy Enhancing Technologies, pp. 46-66. Springer, Berkeley (2001)

4. Stoica, I., Morris, R., Karger, D., Kaashoek, M.F., Balakrishnan, H.: Chord: a scalable peer-to-peer lookup service for internet applications. ACM SIGCOMM Comput. Commun. Rev 31, 149-160 (2001)

5. Ratnasamy, S., Francis, P., Handley, M., Karp, R., Shenker, S.: A scalable content-addressable network. ACM SIGCOMM Comput. Commun. Rev 31, 161-172 (2001)

6. Qiu, T., Chan, E., Ye, M., Chen, G., Zhao, B.Y.: Peer-exchange schemes to handle mismatch in peerto-peer systems. J. Supercomput 48, 15-42 (2009)

7. Liu, Y.: A two-hop solution to solving topology mismatch. IEEE Trans. Parallel Distrib. Syst. 19, 1591-1600 (2008)

8. Liu, Y., Zhuang, Z., Xiao, L., Ni, L.M.: A distributed approach to solving overlay mismatching problem. In: Proceedings of the 24th International Conference on Distributed Computing Systems, pp. 132-139. IEEE Computer Society, Tokyo (2004)

9. Hsiao, H.C., Liao, H., Yeh, P.S.: A near-optimal algorithm attacking the topology mismatch problem in unstructured peer-to-peer networks. IEEE Trans. Parallel Distrib. Syst. 21, 983-997 (2010)

10. Singh, A., Haahr, M.: Decentralized clustering in pure $\mathrm{p} 2 \mathrm{p}$ overlay networks using Schelling's model. In: IEEE International Conference on Communications, pp. 1860-1866. IEEE Computer Society, Glasgow (2007)

11. Rostami, H., Habibi, J.: Topology awareness of overlay P2P networks. Concurr. Comput. Pract. Exp 19, 999-1021 (2007)

12. Leitão, J., Marques, J.P., Pereira, J., Rodrigues, L.: X-bot: a protocol for resilient optimization of unstructured overlay networks. IEEE Trans. Parallel Distrib. Syst. 23, 2175-2188 (2012) 
13. Ren, S., Guo, L., Jiang, S., Zhang, X.: SAT-Match: a self-adaptive topology matching method to achieve low lookup latency in structured p2p overlay networks. In: Parallel and Distributed Processing Symposium, pp. 83-94. IEEE Computer Society, Santa Fe (2004)

14. Qiu, T., Wu, F., Chen, G.: A generic approach to make structured peer-to-peer systems topologyaware. In: Third International Symposium on Parallel and Distributed Processing and Applications, pp. 816-826. Springer, Nanjing (2005)

15. Qiu, T., Chen, G., Ye, M., Chan, E., Zhao, B.Y.: Towards location-aware topology in both unstructured and structured P2P systems. In: International Conference on Parallel Processing, pp. 30-30. IEEE Computer Society, Xian (2007)

16. Liu, Y., Zhuang, Z., Xiao, L., Ni, L.M.: AOTO: Adaptive overlay topology optimization in unstructured P2P systems. In: Global Telecommunications Conference, pp. 4186-4190. IEEE Computer Society, San Francisco (2003)

17. Leitao, J., Marques, J.P., Pereira, J., Rodrigues, L.: X-bot: a protocol for resilient optimization of unstructured overlays. In: Proceedings of the 28th IEEE International Symposium on Reliable Distributed Systems, pp. 236-245. IEEE Computer Society, Niagara Falls (2009)

18. Dumitrescu, M., Andonie, R.: Clustering superpeers in $\mathrm{p} 2 \mathrm{p}$ networks by growing neural gas. In: Proceedings of the 20th Euromicro International Conference on Parallel, Distributed and NetworkBased Processing, pp. 311-318. IEEE Computer Society, Munich (2012)

19. Babaoglu, O., Meling, H., Montresor, A.: Anthill: a framework for the development of agent-based peer-to-peer systems. In: Proceedings of the 22nd International Conference on Distributed Computing Systems, pp. 15-22. IEEE Computer Society, Vienna (2002)

20. Forestiero, A., Leonardi, E., Mastroianni, C., Meo, M.: Self-Chord: a bio-inspired p2p framework for self-organizing distributed systems. IEEE Trans. Netw 18, 1651-1664 (2010)

21. Domic, N.G., Goles, E., Rica, S.: Dynamics and complexity of the schelling segregation model. Phys. Rev. E 83, 96-111 (2011)

22. Thathachar, M., Sastry, P.S.: Networks of Learning Automata: Techniques for Online Stochastic Optimization. Kluwer Academic Publishers, Dordrecht (2004)

23. Lalitha, B., Rao, C.D.S.: GPS based topology matching algorithm for p2p systems. Int. J. Adv. Res. Comput. Sci. Softw. Eng 3, 1-10 (2013)

24. Lalitha, B., Subba Rao, C.H.D.: Mitigation of topology mismatch problem based on node network positioning in unstructured p2p networks. i-manager's J. Comput. Sci 1, 22-32 (2013)

25. Aggarwal, V., Feldmann, A., Scheideler, C.: Can ISPs and p2p users cooperate for improved performance? ACM SIGCOMM Comput. Commun. Rev 37, 29-40 (2007)

26. Ratnasamy, S., Handley, M., Karp, R., Shenker, S.: Topologically-aware overlay construction and server selection. In: Proceedings of the 21st Annual Joint Conference of the IEEE Computer and Communications Societies, pp. 1190-1199. IEEE Computer Society, New York (2002)

27. Tian, R., Xiong, Y., Zhang, Q., Li, B., Zhao, B.Y., Li, X.: Hybrid overlay structure based on random walks. In: Peer-to-Peer Systems IV, pp. 152-162. Springer (2005)

28. Zhang, X.Y., Zhang, Q., Zhang, Z., Song, G., Zhu, W.: A construction of locality-aware overlay network: mOverlay and its performance. IEEE J. Sel. Areas Commun 22, 18-28 (2004)

29. Scheidegger, M., Braun, T.: Improved locality-aware grouping in overlay networks. In: Kommunikation in Verteilten Systemen, pp. 27-38. Springer, Bern (2007)

30. Wolf, S., Merz, P.: Evolutionary local search for the super-peer selection problem and the p-hub median problem. In: Proceedings of the 4th International Conference on Hybrid Metaheuristics, pp. 1-15. Springer, Berlin, Heidelberg (2007)

31. Ju, H.-J., Du, L.-J.: Nodes clustering method in large-scale network. In: Proceedings of the 8th International Conference on Wireless Communications, Networking and Mobile Computing, pp. 1-4. IEEE Computer Society, Shanghai (2012)

32. Li, Y., Yu, Z.: An improved genetic algorithm for network nodes clustering. In: Proceedings of the Second International Conference on Information Computing and Applications, pp. 399-406. Springer Berlin Heidelberg, Qinhuangdao (2011)

33. Jiang, Y., You, J., He, X.: A particle swarm based network hosts clustering algorithm for peer-to-peer networks. In: International Conference on Computational Intelligence and Security, pp. 1176-1179. IEEE Computer Society, Guangzhou (2006)

34. Rostami, H., Habibi, J.: A mathematical foundation for topology awareness of $\mathrm{p} 2 \mathrm{p}$ overlay networks. In: Grid and Cooperative Computing, pp. 906-918. Springer, Heidelberg (2005)

35. Liu, Y., Xiao, L., Liu, X., Ni, L.M., Zhang, X.: Location awareness in unstructured peer-to-peer systems. IEEE Trans. Parallel Distrib. Syst. 16, 163-174 (2005) 
36. Hsiao, H.C., Liao, H., Huang, C.C.: Resolving the topology mismatch problem in unstructured peerto-peer networks. IEEE Trans. Parallel Distrib. Syst. 20, 1668-1681 (2009)

37. Papadakis, H., Fragopoulou, P., Markatos, E., Roussopoulos, M.: ITA: innocuous topology awareness for unstructured p2p networks. IEEE Trans. Parallel Distrib. Syst. 24, 1589-1601 (2013)

38. Xiao, L., Liu, Y., Ni, L.M.: Improving unstructured peer-to-peer systems by adaptive connection establishment. IEEE Trans. Comput. 54, 1091-1103 (2005)

39. Yunhao, L., Xiao, L., Ni, L.M.: Building a scalable bipartite P2P overlay network. IEEE Trans. Parallel Distrib. Syst. 18, 1296-1306 (2007)

40. Saghiri, A.M., Meybodi, M.R.: A distributed adaptive landmark clustering algorithm based on mOverlay and learning automata for topology mismatch problem in unstructured peer-to-peer networks. Int. J. Commun Syst (2015). doi:10.1002/dac.2977

41. Baumgart, I., Heep, B., Krause, S.: OverSim: A scalable and flexible overlay framework for simulation and real network applications. In: Peer-to-Peer Computing. pp, 87-88. IEEE Computer Society, Seattle, Washington (2009)

42. Baumgart, I., Heep, B., Krause, S.: OverSim: A flexible overlay network simulation framework. In: IEEE Global Internet Symposium, pp. 79-84. IEEE Computer Society, Anchorage (2007)

43. Baumgart, I., Gamer, T., Hübsch, C., Mayer, C.P.: Realistic underlays for overlay simulation. In: Proceedings of the 4th International ICST Conference on Simulation Tools and Techniques, pp. 402-405. ICST (Institute for Computer Sciences, Social-Informatics and Telecommunications Engineering), Brussels (2011)

44. Li, L., Alderson, D., Willinger, W., Doyle, J.: A first-principles approach to understanding the internet's router-level topology. ACM SIGCOMM Comput. Commun. Rev 34, 3-14 (2004)

45. Ripeanu, M.: Peer-to-peer architecture case study: Gnutella network. In: First International Conference on Peer-to-Peer Computing, pp. 99-100. IEEE Computer Society, Linkoping (2001)

46. Liu, Y., Xiao, L., Esfahanian, A.H., Ni, L.M.: Approaching optimal peer-to-peer overlays. In: Proceedings of the 13th IEEE International Symposium on Modeling, Analysis, and Simulation of Computer and Telecommunication Systems, pp. 407-414. IEEE Computer Society, Atlanta (2005)

47. SNAP: Network datasets: Gnutella peer-to-peer network, http://snap.stanford.edu/data/p2pGnutella04.html

48. Mahadevan, P., Krioukov, D., Fomenkov, M., Huffaker, B., Dimitropoulos, X., Vahdat, A.: Lessons from Three Views of the Internet Topology. University of California, San Diego (2005)

49. Huffaker, B., Plummer, D., Moore, D., Claffy, K.C.: Topology discovery by active probing. In: Proceedings of Symposium on Applications and the Internet Workshops, pp. 90-96., Washington (2002)

50. Narendra, K.S., Thathachar, M.A.L.: Learning Automata: An Introduction. Prentice-Hall, Englewood Cliffs (1989)

Ali Mohammad Saghiri received the B. Sc. and M. Sc. degrees in computer engineering in Iran, in 2008 and 2010, respectively. He is currently the Ph.D. student of computer engineering in AmirKabir University of Technology, Tehran, Iran. His research interests include distributed systems, artificial intelligence, and bio-inspired computation.

Mohammad Reza Meybodi received the B.S. and M.S. degrees in Economics from Shahid Beheshti University in Iran, in 1973 and 1977, respectively. He also received the M.S. and Ph.D. degree from Oklahoma University, USA, in 1980 and 1983, respectively in Computer Science. Currently, he is a full professor in Computer Engineering Department, Amirkabir University of Technology, Tehran, Iran. His research interests include learning systems, parallel algorithms, and soft computing. 